American Journal of Biochemistry and Biotechnology 7 (4): 146-162, 2011

ISSN 1553-3468

(C) 2011 Ghaly and Al hattab et al., This open access article is distributed under a Creative Commons Attribution

(CC-BY) 3.0 license

\title{
Effect of Diurnally Cyclic Temperature on the Performance of a Continuous Mix Anaerobic Digester
}

\author{
Abdel E. Ghaly and Mariam T. Al hattab \\ Department of Process Engineering and Applied Sciences, \\ Faculty of Engineering, Dalhousie University Halifax, Nova Scotia, Canada
}

\begin{abstract}
Problem statement: Concentrated livestock production is a major contributor to environmental problems through the production of large quantity of husks on relatively small land area. Anaerobic digestion represents a valuable means of cover by these husks into methane while reducing manure pollution problems. Methodology: An experimental apparatus, including data acquisition and control system, was developed and used to investigate the effects of two diurnally cyclic temperature ranges $\left(20-40\right.$ and $\left.15-25^{\circ} \mathrm{C}\right)$ and four levels of hydraulic retention times $(25,20,15$ and $10 \mathrm{~d}$ ) on the performance of anaerobic reactors operated on screened dairy manure. Results: The reactor temperature exhibited a lag relative to the chamber temperature. For the $20-40^{\circ} \mathrm{C}$ temperature cycles, the average lag at the maximum chamber temperature was $3.75 \mathrm{~h}$ while the lag at the minimum chamber temperature was $4.37 \mathrm{~h}$. For the $15-25^{\circ} \mathrm{C}$ temperature cycle, the average lag at maximum chamber temperatures was 3.61 while the lag of the minimum chamber temperature was $4.34 \mathrm{~h}$. The effluent solids content, total Kjeldahl nitrogen and ammonium nitrogen concentrations were not adversely affected by the reactor diurnally cyclic temperature. The observed values of these parameters compared fairly well with the values obtained by other researchers. The effluent total solids, volatile suspended solids, $\mathrm{TKN}, \mathrm{NH}_{4}-\mathrm{N}$ and methane content of the biogas diurnally cyclic patterns were out of phase with the diurnally cyclic pattern of the reactor temperature by about $12 \mathrm{~h}$ under most of the investigated operating conditions. The $\mathrm{pH}$ and the carbon dioxide content of the biogas all exhibited a diurnally cyclic pattern which was in phase with the reactor diurnally cyclic temperature. The reductions in total solids, total suspended solids, fixed suspended solids, total Kjeldahl nitrogen, ammonium nitrogen and methane yield were all significantly affected by the diurnal temperature range and hydraulic retention time. Conclusion: The measured reactor $\mathrm{pH}$ followed a diurnally cyclic pattern which was in phase with the reactor temperature. At the operating temperature cycle of $20-40^{\circ} \mathrm{C}$, the reactor $\mathrm{pH}$ ranged from 6.80-7.54 while VFA concentration ranged from $44.7-154.8 \mathrm{mg} \mathrm{L}^{-1}$. At the operating temperature cycle of $15-25^{\circ} \mathrm{C}$, the $\mathrm{pH}$ ranged from $6.30-6.60$ while the VFA concentration ranged from $151.0-1187.0 \mathrm{mg} \mathrm{L}^{-1}$.
\end{abstract}

Key words: Anaerobic digestion, dairy manure, continuous mix, diurnal temperature, solids, nitrogen, volatile fatty acids, biogas, methane, livestock production

\section{INTRODUCTION}

Concentrated livestock production is a major contributor to environmental pollution problems through the production of large quantities of wastes on a relatively small land area. When exposed to surface waters, animal manure leads to: (a) an over-enrichment of nutrients causing eutrophication problems (Mogensen et al., 2009), (b) high organic matter content resulting in the exertion of high Biochemical Oxygen Demand (BOD) and causing an ecological imbalance (Dieu, 2009), (c) high pathogen content causing numerous human and animal diseases (Pimentel et al., 2008a) and (d) high air contamination by obnoxious odorous compounds causing widespread health problems (Bradford et al., 2009). In addition, when manure is decomposed under anaerobic conditions, it releases heat trapping gases such as methane $\left(\mathrm{CH}_{4}\right)$ and Nitrous Oxide $\left(\mathrm{N}_{2} \mathrm{O}\right)$ which contribute to climate change (Izaurralde, 2009).

Modern systems of farming have also resulted in an increased demand of energy. Efforts to increase the quantity of food produced, to meet the needs of a growing population, have led to large mechanical

Corresponding Author: Abdel Ghaly, Department of Process Engineering and Applied Sciences, Faculty of Engineering, Dalhousie University Halifax, Nova Scotia, Canada 
energy inputs and increased use of fertilizers and other chemicals which resulted in an increased competition for available energy supplies (Pimentel and Pimentel, 2008). Unfortunately, man has depended for a long time on the burning of finite and non-renewable fossil fuels for meeting his energy needs (Andres et al., 1999). It was not until the energy crises, caused by the wars in the Middle East in the 1970s and 1990s which clearly dramatized the vulnerability of a fossil-fuel based economy, that serious awareness was created over the need for intensified effort at finding alternative energy sources (Cleveland, 1995). Among the various alternative energy sources, biomass (a general term that refers to plant and animal materials) is perhaps the only energy source which is available and affordable to the third world population (Legros et al., 2009). Many chemical and biological conversion processes are available for the release of energy stored in these materials. Among them, anaerobic digestion represents a valuable means of converting biomass to methane (a more efficient and versatile fuel) while acting as a vital means of reducing animal manure pollution problems (Macias-Corral et al., 2008; Riano et al., 2011).

Anaerobic digestion is a biological process in which organic materials are decomposed in the absence of free oxygen to yield methane $\left(\mathrm{CH}_{4}\right)$, carbon dioxide $\left(\mathrm{CO}_{2}\right)$ and small quantities of other gases $\left(\mathrm{H}_{2} \mathrm{~S}, \mathrm{~N}_{2}\right.$ and $\mathrm{H}_{2} \mathrm{O}$ ). The process requires the concerted actions of a symbiotic population of three groups of facultative and obligate anaerobic bacteria to: (a) degrade carbohydrates, lipids and proteins into alcohols and long chain fatty acids, (b) produce acetate, carbon dioxide and hydrogen from the end products of the fermentation of organic materials and (c) produce methane and carbon dioxide from the fermentation of acetate as shown in Fig. 1 (Ghaly et al. 2000).

Compared to other biological treatment processes, anaerobic digestion has several advantages including: (a) production of usable biogas that is about $60-80 \%$ methane with a fuel value of 17-23.9 $\mathrm{MJ} \mathrm{m}^{-3}$ (MaciasCorral et al., 2008; Pimentel et al., 2008b; Porpatham et al., 2008), (b) the digested residue is almost odourless with reduced solids content (Burke, 2001; Dieu, 2009), (c) the inorganic nutrients are conserved in the digestion process resulting in the enhancement of the fertilizer value of the digested sludge (Iannotti et al., 1979) and (d) pathogenic microorganisms such as Salmonella Sp. and Brucella $\mathrm{Sp}$. as well as weed seeds are destroyed during the anaerobic digestion process (Pyle, 1978; Jeyanayagam and Collins, 1984; ElMashad et al., 2003; Masse et al., 2011). The latter implies that livestock can be grazed on pastures which have been spread with sludge from the anaerobic digester sooner than would be acceptable if raw manure was used (Blanchard and Gill, 1987).

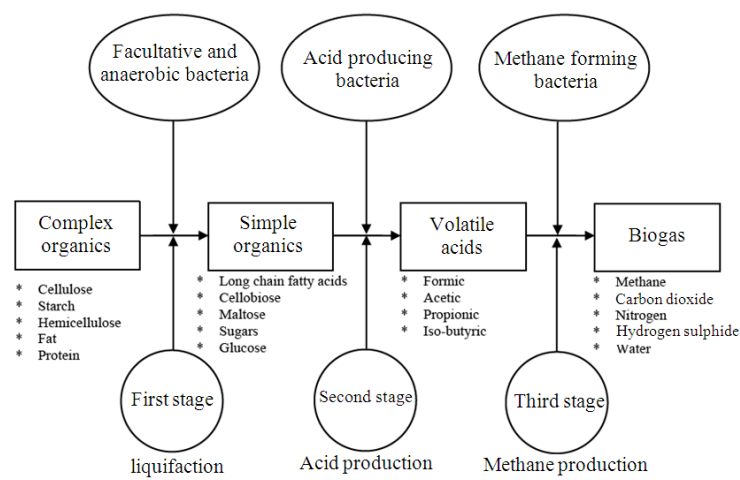

Fig. 1: Three stage anaerobic digestion process

Despite these advantages, anaerobic digestion has not enjoyed widespread acceptance among many farmers due to the relative instability of the system resulting from the sensitivity of anaerobic bacteria to adverse environmental conditions (Ghaly et al., 2000). In particular, temperature is considered to be one of the most important environmental parameters affecting the growth and survival of anaerobic microorganisms (Chen and Day, 1986; Parkin and Owen, 1986; Angelidaki and Ahring, 1994; Burke, 2001; El-Mashad, 2004; Kim et al., 2006; Chae et al., 2008; Appels et al., 2010; Lianhu et al., 2010; Gustin and Marinsek-Logar, 2011). However, the effect of gradual variation in temperature in a diurnal cycle is yet to be investigated.

The aim of this study was to investigate the performance of a continuous mix anaerobic reactor operating under diurnally cyclic temperatures by evaluating reductions in the effluent solids and nitrogenous compounds and the profile of VFA.

\section{MATERIALS AND METHODS}

Experimental apparatus: The experimental apparatus (Fig. 2) consisted of four bioreactors, a feeding system, a temperature control system, a gas collection system, a data acquisition and control system and computer.

Each bioreactor (Fig. 3) was constructed of a cylindrical PVC vessel, (458 mm length, $300 \mathrm{~mm}$ diameter and a $10 \mathrm{~mm}$ wall thickness) it provided a liquid volume of $25 \mathrm{~L}$ and a gas head space of $7.4 \mathrm{~L}$. The cover of the bioreactor was made of a transparent Plexiglas plate $(370 \mathrm{~mm}$ diameter and $10 \mathrm{~mm}$ thickness). It was secured to the bioreactor, through PVC lugs glued onto the top outer periphery of the vessel, by means of six bolts. A Vaseline-coated rubber gasket was fitted between the cover and the vessel to provide a gas-tight seal. The temperature and $\mathrm{pH}$ 
sensors were mounted on special holders threaded through the reactor cover and immersed to a depth of $100 \mathrm{~mm}$ below the liquid surface. The contents of the bioreactor were stirred by means of a flat-bladed impeller driven by a 1/12 HP electric motor (Model No. 6105061401, Franklin, Bluffton, Indiana, USA) at a speed of $125 \mathrm{rpm}$. The manure feed inlet consisted of a $25 \mathrm{~mm}$ diameter PVC tube inserted through the bioreactor cover to a depth of $246 \mathrm{~mm}$ below the liquid surface. The effluent port was located opposite to the feed inlet port at a distance of $300 \mathrm{~mm}$ above the bottom of the bioreactor.

The manure feeding system consisted of a manure storage tank, a feed pump, a set of tubing, a distribution manifold and a set of solenoid valves. A $60 \mathrm{~L}$ plastic container was used to store the manure. It was fitted with a stirring paddle driven by a $1 / 12 \mathrm{HP}$ electric motor (Model 55CP10FG17AX, General Electric, Missisauga, Ontario, Canada) mounted on the tank cover. A feeding port sealed with a rubber bung as well as a ventilation port were provided on the tank cover. The outlet port of the tank was located at the lower portion of the tank side. A manual valve was provided to control the flow of manure from the tank. A peristaltic pump (Model 110-030, TAT Engineering Co., Logan, Ohio, USA) was used to feed the manure. At a rotational speed of $2 \mathrm{rpm}$ and an output of 0.138 $\mathrm{L}$ of manure per revolution, the pump delivered the manure at a capacity of $16.56 \mathrm{~L} \mathrm{~h}^{-1}$ from the storage tank through the valve, tygon tubing, the distribution manifold and a set of automatically controlled solenoid valves to the individual reactors. A digital timer controlled and synchronized the operation of the feed tank stirrer (Model 5935932, Type NSI10R93, Bodine Electric Co, North York, Ontario, Canada), the feed pump and the solenoid valves to ensure the delivery of a well mixed manure to the bioreactors at a predetermined sequence and loading rate. Eight feeding cycles were carried out daily at an interval
3

$\mathrm{h}$.

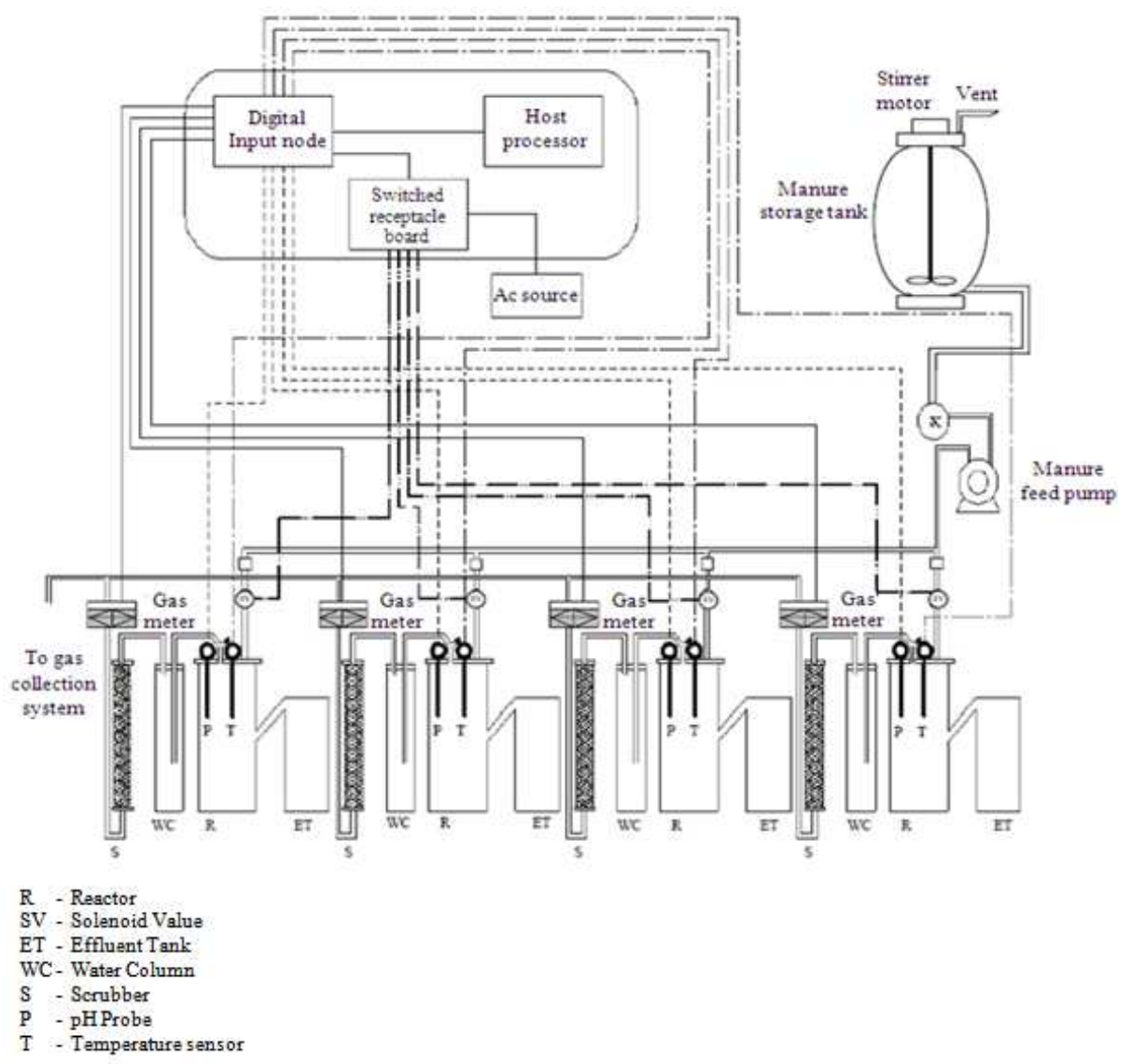

Fig. 2: Schematic of the experimental apparatus 
Am. J. Biochem. \& Biotech., 7 (4): 146-162, 2011

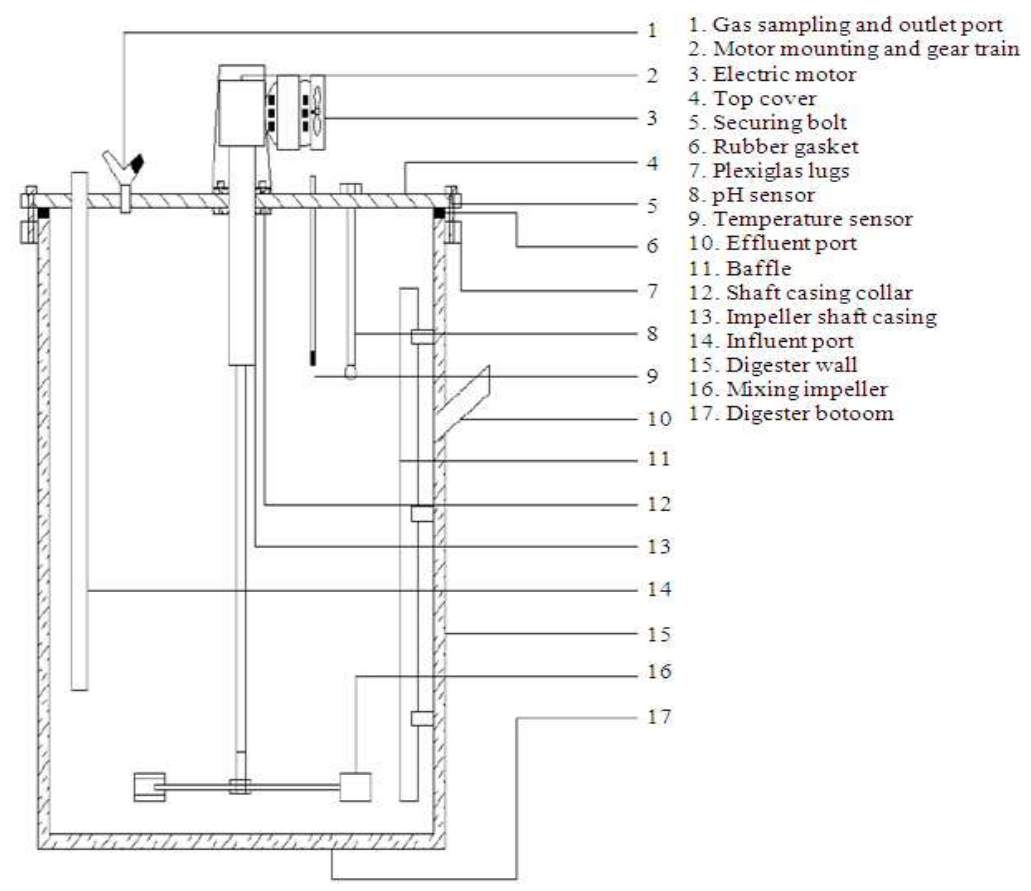

Fig. 3: The continuous mix anaerobic bioreactor

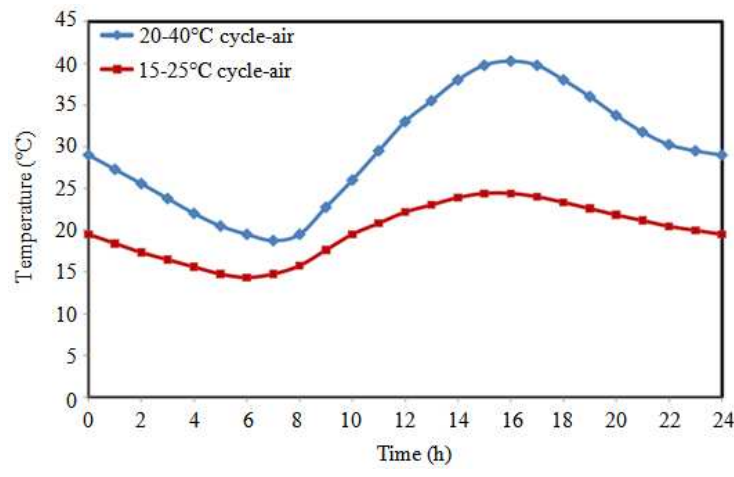

Fig. 4: Diurnal temperature profiles

The objective of the temperature control system was to maintain the profiles of the two temperature cycles (Fig. 4) in the enclosed chamber in which the digesters were located. It consisted of an insulted chamber, a chiller, two heaters, two cross flow heat exchangers, two fans, temperature transducers and ducts for directing the circulation of air (Fig. 5). The chamber was made of a $20 \mathrm{~mm}$ thick wooden box $(2440 \mathrm{~mm}$ long, $710 \mathrm{~mm}$ wide and $525 \mathrm{~mm}$ deep) and divided into two compartments each was padded with $40 \mathrm{~mm}$ of Styrofoam insulation (R-value of $0.88 \mathrm{~m}^{2} \mathrm{~K} / \mathrm{W}$ ). Each compartment was maintained at one of the two temperature profiles and contained two bioreactors.
On the floor of each compartment was a U-shaped galvanized steel duct of $100 \mathrm{~mm}$ inside diameter. A fan, a heat exchanger and a heater were located (in that order) inside one arm of each duct which circulated air in a closed loop. The fan drew air from the chamber into the duct and the air circulated over the heat exchanger and the heater and then left the duct through perforations on the other two sections of the U-shaped duct to bathe the bioreactor. The air was circulated using a thermally protected electric fan (Model 4C02A, Dayton Electric Manufacturing. Co., Niles, Illinois, USA). The capacities of the heaters were 750 and $500 \mathrm{~W}$ for the compartments operated at the higher and lower cyclic temperature ranges, respectively. A digital rapid cool refrigerated bath (Series 900, Polyscience, Niles, Illinois, USA) with an operating temperature range of $-35-150^{\circ} \mathrm{C}$ and an accuracy of $\pm 0.02^{\circ} \mathrm{C}$ was used. The chiller had a maximum cooling capacity of $425 \mathrm{~W}$ at $20^{\circ} \mathrm{C}$ (or 225 $\mathrm{W}$ at $-10^{\circ} \mathrm{C}$ ), a bath capacity of $13 \mathrm{~L}$ and can deliver a maximum flow of $15 \mathrm{~L} \mathrm{~min}^{-1}$ at zero head. Two submersible pumps (Model 1-MA, Little Giant, Bluffton, Indiana, USA), each delivered chilled water to one of the two heat exchangers, were immersed inside the chiller bath. With a power consumption of $3.73 \mathrm{~W}$, each pump had a rated capacity of $10.72 \mathrm{~L} \mathrm{~min}^{-1}$ at $0.3 \mathrm{~m}$ and a maximum pumping height of $1.85 \quad \mathrm{~m}$. 


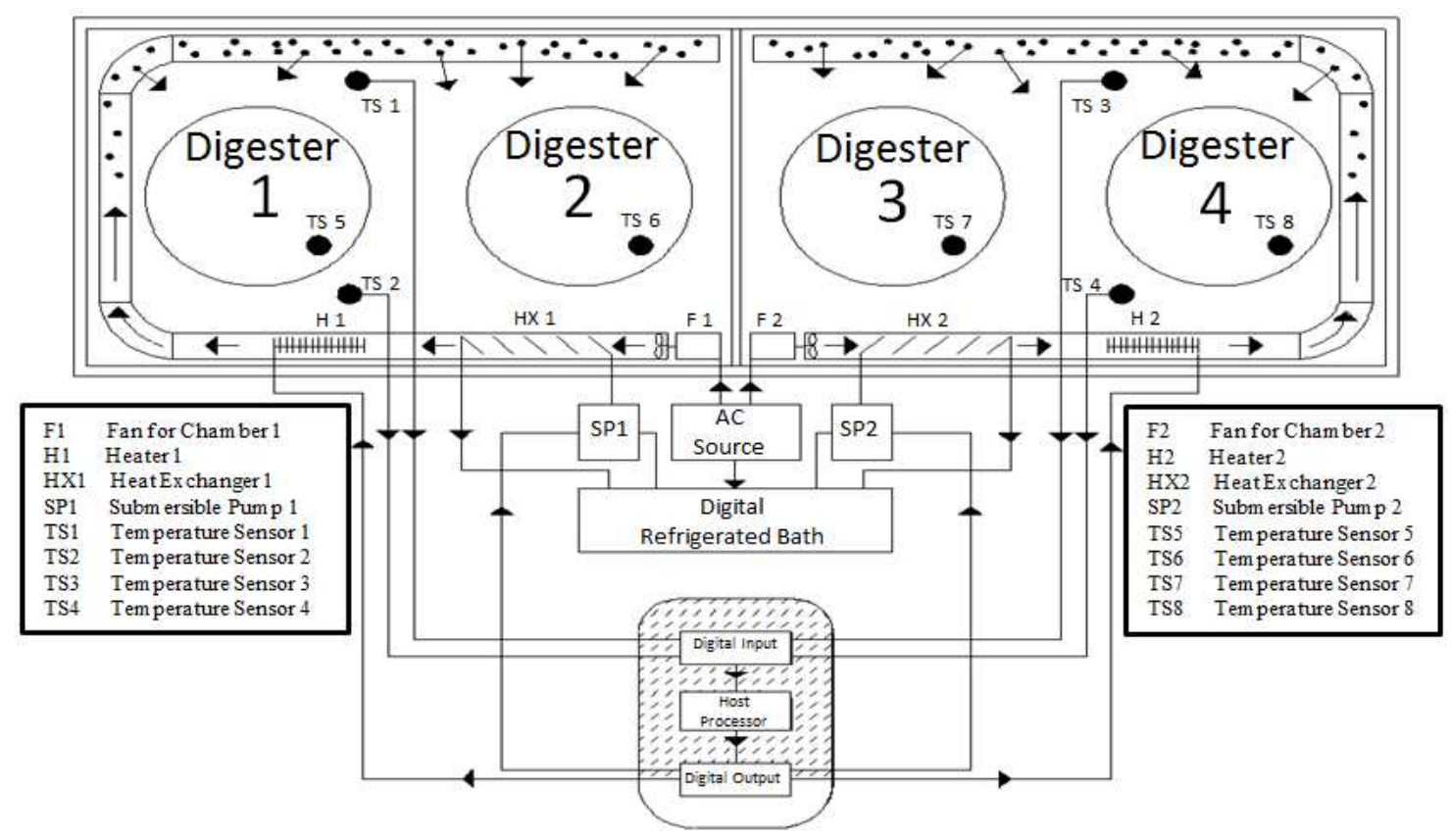

Fig. 5: The temperature control system

A Styrofoam cover of the same thickness as the wall insulation was provided for each of the chamber compartments in such a way that visual examination of the content of the reactors was possible without removing the covers. Four Temperature Sensors (TS1 and TS2 and TS3 and TS4) were mounted on the chamber covers (two for each compartment). During operation, the temperature transducers continuously sampled the air temperature in each of the chamber compartments and transmitted the signals through the digital input node of the data acquisition system to the host processor. Every $30 \mathrm{sec}$, the host processor computed the average temperature of each compartment and compared these with set point values. Depending on the error signal, the host processor generated a correction signal which either turned the heaters and the chilled water submersible pumps on and off (or vice versa), respectively.

Gas collection was effected through a Y-shaped nipple fitted on the cover. One end of the nipple carried a rubber septum through which gas samples were drawn with a syringe. The other arm of the nipples was fitted with flexible tubing which conveyed the evolved gases through a water column, gas scrubber and gas meter to the gas collection and storage system. The water column was used to provide back pressure in the reactor to maintain a constant liquid level. The scrubber was made of a gas-tight Plexiglas column $(80 \mathrm{~mm}$ diameter and $450 \mathrm{~mm}$ long) filled with steel wool to strip the biogas of hydrogen sulphide. The gas meter was a tipping balance meter which indicated when $50 \pm 2 \mathrm{~mL}$ of gas had been collected. The collected gas was subsequently pumped into a gas cylinder by means of a compressor.

Manure collection, preparation and storage: Dairy manure was collected from a commercial dairy farm located in Stewiacke, Nova Scotia, Canada. The manure was scrapped off the floor of the dairy barn and screened using a modified fish de-boner (Model SDX16, Bibun, Japan). During the sieving process, tap water was added to dilute the manure to $6.5 \%$ solid content. The manure was placed in buckets of $30 \mathrm{~L}$ and the buckets were sealed and transported to a commercial freezing plant (Associated Freezers, Dartmouth, Nova Scotia, Canada) where they were stored at $-25^{\circ} \mathrm{C}$ until needed. When needed, buckets of manure were removed from the freezer and kept at room temperature in the Waste Management Laboratory to thaw for $48 \mathrm{~h}$ and the manure was then loaded into the feeding tank. Some characteristics of raw manure are shown in Table 1.

Start-up procedure: The bioreactors were started by adding $18.0 \mathrm{~L}$ (to each reactor) of actively digesting sewage sludge obtained from Mill Cove Municipal Wastewater Treatment Plant (Bedford, Nova Scotia, Canada). This was followed by the addition of $7.0 \mathrm{~L}$ of 
screened dairy manure. The temperature control and the data acquisition systems were activated and the digesters were operated in batch mode for $48 \mathrm{~h}$. The feeding system was used to feed the reactors. Reactors R1 and $\mathrm{R} 2$, were operated at the diurnal temperature cycle of 20$40{ }^{\circ} \mathrm{C}$ and received feed at rates of 0.33 and $0.42 \mathrm{~L} \mathrm{~d}^{-1}$, respectively. Reactors $\mathrm{R} 3$ and $\mathrm{R} 4$ were operated at the diurnal temperature cycle of $15-25^{\circ} \mathrm{C}$ and received feed at rates of 0.33 and $0.42 \mathrm{~L} \mathrm{~d}^{-1}$, respectively. Feeding rates were equivalent to hydraulic retention times (HRTs) of 75 days for the feeding rate of $0.33 \mathrm{~L} \mathrm{~d}^{-1}$ and 60 days for the feeding rate of $0.42 \mathrm{~L} \mathrm{~d}^{-1}$. The feeding rates were then adjusted to $0.5 \mathrm{~L} \mathrm{~d}^{-1}$ for reactors $\mathrm{R} 1$ and $\mathrm{R} 3$ and $0.63 \mathrm{~L} \mathrm{~d}^{-1}$ for $\mathrm{R} 2$ and $\mathrm{R} 4$ and were held constant for $72 \mathrm{~h}$. These rates corresponded to HRTs of 50 and 40 days, respectively. These rates were maintained for another $48 \mathrm{~h}$.

Operating procedure: After 7 days from start-up, R1 and R3 were operated at an HRT of 25 days while R2 and R4 were operated at an HRT of 20 days. The startup period was concluded after a period of 32 days. Following the initial start-up period, monitoring of the biogas production was started on day 33 (from the start). Steady state was construed to have been achieved when a uniform gas production and/or uniform effluent quality were achieved. Once the steady-state was achieved at a given retention time, the system parameters were measured/monitored for a period of at least five days. The feeding rates of the reactors were adjusted for the next set of retention times (15 days for reactors $\mathrm{R} 1$ and $\mathrm{R} 3$ and 10 days for reactors $\mathrm{R} 2$ and R4). When the steady state was attained, sampling/monitoring was continued for five days.

Table 1: Characteristics of raw manure

\begin{tabular}{lr}
\hline Parameter & Mean value* \\
\hline Total solids $(\mathrm{g} / \mathrm{L})$ & 64.25 \\
Total volatiles solids $(\mathrm{g} / \mathrm{L})$ & 50.26 \\
(\% of total solids) & 78.23 \\
Total fixed solids $(\mathrm{g} / \mathrm{L})$ & 13.99 \\
Total suspended solids $(\mathrm{g} / \mathrm{L})$ & 42.25 \\
Volatile suspended solids $(\mathrm{g} / \mathrm{L})$ & 31.00 \\
Fixed suspended solids $(\mathrm{g} / \mathrm{L})$ & 11.25 \\
Total COD $(\mathrm{g} / \mathrm{L})$ & 98.80 \\
Soluble COD $(\mathrm{g} / \mathrm{L})$ & 27.90 \\
Total kjeldahl nitrogen $(\mathrm{g} / \mathrm{L})$ & 3.80 \\
Ammonium nitrogen $(\mathrm{g} / \mathrm{L})$ & 2.45 \\
NH $4-\mathrm{N} / \mathrm{TKN}$ ratio & 0.64 \\
Volatile FATTY ACID $(\mathrm{VFA})$ & \\
Acetic $(\mathrm{g} / \mathrm{L})$ & 1.55 \\
Propionic $(\mathrm{g} / \mathrm{L})$ & 0.28 \\
i-Butyric $(\mathrm{g} / \mathrm{L})$ & 0.04 \\
Butyric $(\mathrm{g} / \mathrm{L})$ & 0.06 \\
i-Valeric $(\mathrm{g} / \mathrm{L})$ & 0.04 \\
Valeric $(\mathrm{g} / \mathrm{L})$ & 0.02 \\
i-Caprioc $(\mathrm{g} / \mathrm{L})$ & 0.01 \\
Caprioc $(\mathrm{g} / \mathrm{L})$ & 0.01 \\
Heptanoic $(\mathrm{g} / \mathrm{L})$ & 0.04 \\
Total VFA as Acetic acid $(\mathrm{g} / \mathrm{L})$ & 1.91 \\
\hline * Each value represents the average of 5 samples
\end{tabular}

Analyses: The liquid samples were analyzed for Total Solids (TS), Total Volatile Solids (TVS), Total Fixed Solids (TFS), Total Suspended Solids (TSS), Volatile Suspended Solids (VSS), Fixed Suspended Solids (FSS), Total Kjeldahl Nitrogen (TKN), ammonium nitrogen $\left(\mathrm{NH}_{4}-\mathrm{N}\right)$ and Volatile Fatty Acids (VFA). The biogas samples were analyzed for gas composition.

The total solids and suspended solids analyses were performed according to procedures described in the Standard Method for the Examination of Water and Wastewater (APHA, 1985). The total Kjeldahl and ammonium nitrogen were determined using a Tecator Kjeltec Auto Analyzer (Model 1030 and Se catalyst, Tecator, Paris, France).

The individual volatile acids $\left(\mathrm{C}_{2}-\mathrm{C}_{7}\right)$ contained in a sample were determined using a Hewlett-Packard gas chromatograph (Model 5890 series II, Mississauga, Ontario, Canada) equipped with an HP 76734A automatic injector. Extraction of the VFA was carried out by acidifying $3.0 \mathrm{~mL}$ of each of the manure samples using $0.1 \mathrm{~mL} 30 \%$ sulphuric acid. The acidified samples were well mixed and centrifuged at $7000 \mathrm{rpm}$ for $20 \mathrm{~min} .2 .0 \mathrm{~mL}$ of the supernatants were decanted and an equal amount of diethyl ether was added. The mixtures were well shaken and then centrifuged at 5000 rpm for $5 \mathrm{~min}$ to break down the emulsion layer. The upper layer which consisted of di-ethyl ether was removed for analysis. Volatile acids were, also, extracted from a volatile acid standard mixture (No46975, SupelCo, Oakville, Ontario, Canada) using diethyl ether. The chromatograph was calibrated by injecting $1.0 \mathrm{~mL}$ of the extracted standard VFA mixture into the $25 \times 0.2 \mathrm{~mm}$ capillary column of the liquid chromatograph whose film thickness is $0.33 \mathrm{~mm} .1 .0$ $\mathrm{mL}$ of the extracted samples were injected into the column. A split ratio of 1:5 was applied. The column temperature was first maintained at $60^{\circ} \mathrm{C}$ for $3 \mathrm{~min}$ and then increased at a rate of $10^{\circ} \mathrm{C} \mathrm{min}^{-1}$ until a temperature of $150^{\circ} \mathrm{C}$ was attained. The column temperature was maintained at $150^{\circ} \mathrm{C}$ for $2 \mathrm{~min}$. The injector was set to $180^{\circ} \mathrm{C}$ while the flame ionization detector was set at $250^{\circ} \mathrm{C}$. The carrier gas was helium at a flow rate of $1.2 \mathrm{~mL} \mathrm{~min}^{-1}$.

The composition of biogas was determined using a gas chromatograph (Model HP 5980A, Hewlett Packard, Mississauga, Ontario, Canada). Samples of 0.1 $\mathrm{mL}$ were taken from the gas collected in the sampling tube using a gas tight locked syringe. The samples were injected into $152.4 \mathrm{~mm} \times 3.2 \mathrm{~mm}(6 \mathrm{in} \times 1 / 8 \mathrm{in}) \mathrm{OD}$ porapak Q stainless steel column of the gas chromatograph which is connected in a series bypass arrangement with a $152.4 \mathrm{~mm} \times 3.2 \mathrm{~mm}(6 \mathrm{in} \times 1 / 8$ in $)$ OD molecular sieve 5 A 60180 stainless steel column. The switch valve of the gas chromatograph was adjusted to permit the molecular sieve column to store 
nitrogen, methane and carbon monoxide until the elution of the $\mathrm{CO}_{2}, \mathrm{C}_{2} \mathrm{H}_{2}$ and $\mathrm{C}_{6} \mathrm{H}_{6}$ through the porapak $\mathrm{Q}$ stainless steel column. The column was maintained at $45^{\circ} \mathrm{C}$ with helium as the carrier gas at $30 \mathrm{~mL} \mathrm{~min}^{-1}$. The injector was set at $150^{\circ} \mathrm{C}$ while the thermal conductivity detector was set at $250^{\circ} \mathrm{C}$.

\section{RESULTS}

Temperature: Typical variations of reactor temperatures with those of the chambers for the 20-40 and $15-25^{\circ} \mathrm{C}$ diurnal temperature cycles are shown in Fig. 6. A summary of the reactor temperature profiles at the various operating conditions is presented in Table 2.

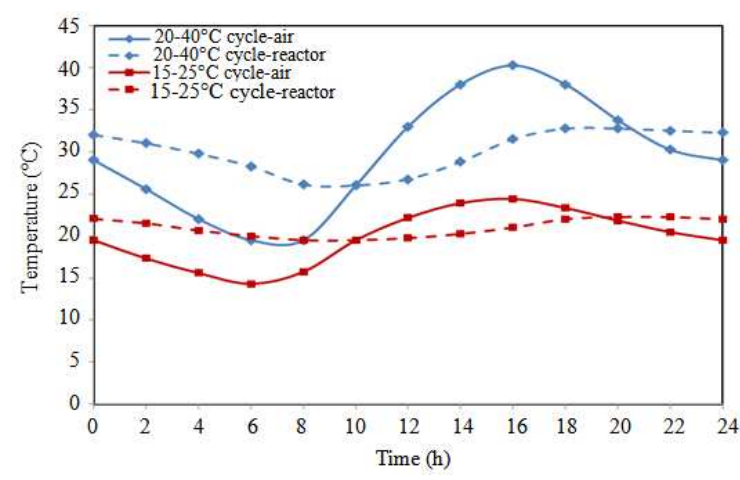

Fig. 6: Diurnal variations in bioreactor and ambient temperatures

Total solids: The average values of total, fixed and volatile solids concentrations of the effluent samples collected during the steady state and their reductions are shown in Table 3. The diurnal variations in the total solids of the effluent collected over a $24 \mathrm{~h}$ period during the steady state are shown in Fig. 7.

Suspended solids: The total suspended, fixed and volatile solids of the effluent samples collected at steady state and their reduction are shown in Table 4. The diurnal variations in suspended solids are shown in Fig. 8.

Nitrogen: The Total Kjeldahl Nitrogen (TKN) and the ammonium nitrogen $\left(\mathrm{NH}_{4}-\mathrm{N}\right)$ concentrations of the effluent samples collected at steady state and their reductions are shown in Table 5. Diurnally cyclic variations in the TKN were observed at all HRTs under both temperature cycles (Fig. 9).

Volatile fatty acids: The concentrations of Volatile Fatty acids (VFAs) in the effluent samples taken during the steady state conditions are shown in Table 6. The VFAs included: acetic, propionic, isobutyric, isovaleric, valeric, iso-caprioc and heptanoic acids.

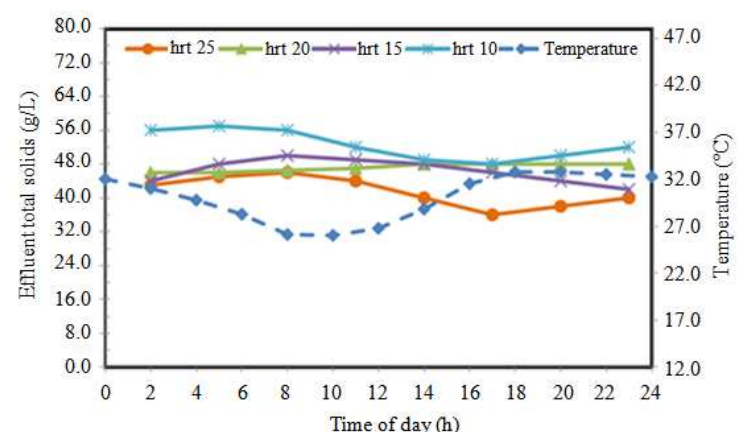

(a) $20-40^{\circ} \mathrm{C}$ temperature cycle

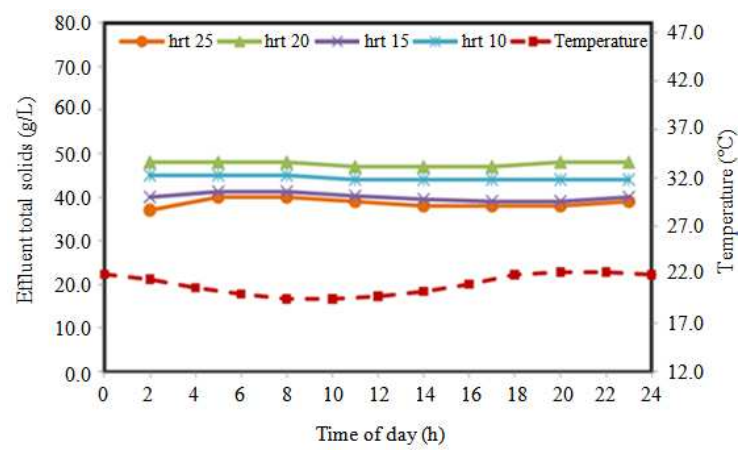

(b) $15-25^{\circ} \mathrm{C}$ temperature cycle

Fig. 7: Diurnal variations in total solids

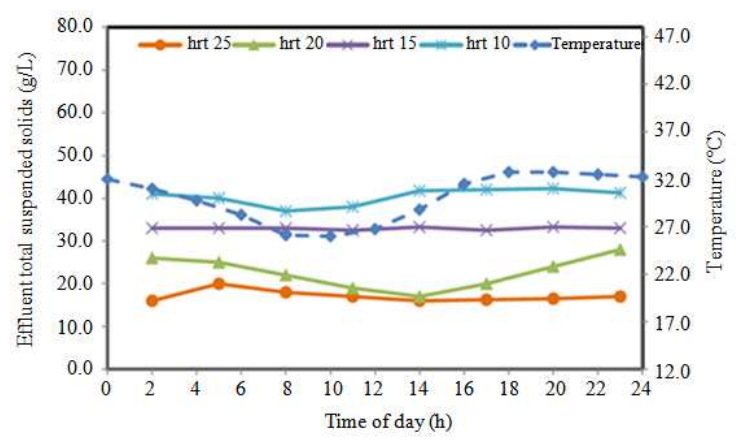

(a) $20-40^{\circ} \mathrm{C}$ temperature cycle

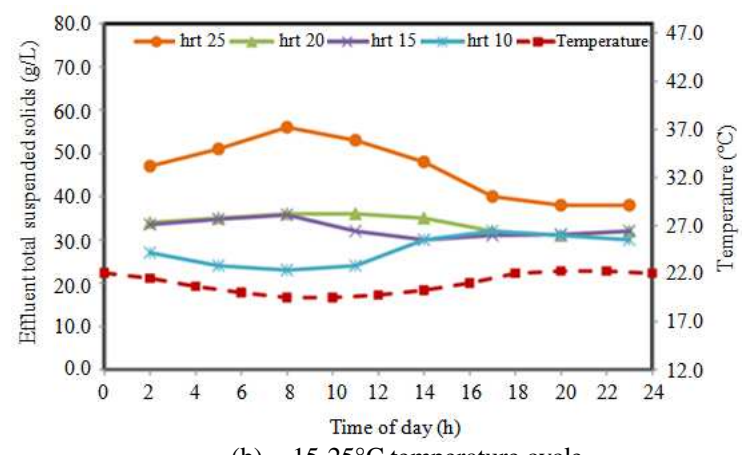

(b) $15-25^{\circ} \mathrm{C}$ temperature cycle

Fig. 8: Diurnal variations in total suspended solids 
Am. J. Biochem. \& Biotech., 7 (4): 146-162, 2011

Table 2: Summary of reactor temperature profiles

\begin{tabular}{|c|c|c|c|c|c|c|}
\hline \multirow{2}{*}{$\begin{array}{l}\text { Temperature } \\
\text { Cycle } \\
\left({ }^{\circ} \mathrm{C}\right)\end{array}$} & \multirow[b]{2}{*}{$\begin{array}{l}\text { HRT } \\
\text { (d) }\end{array}$} & \multicolumn{3}{|c|}{ Reactor Temperature $\left({ }^{\circ} \mathrm{C}\right)$} & \multicolumn{2}{|c|}{ Lag at (h) } \\
\hline & & $\mathrm{T}_{\min }$ & $\mathrm{T}_{\max }$ & A & $\mathrm{L}_{\min }$ & $\mathrm{L}_{\max }$ \\
\hline \multirow[t]{7}{*}{$20-40$} & 25 & 25.50 & 33.70 & 8.20 & 3.38 & 4.35 \\
\hline & 20 & 25.10 & 33.60 & 8.40 & 3.30 & 4.69 \\
\hline & 15 & 26.00 & 33.90 & 7.90 & 3.37 & 3.19 \\
\hline & 10 & 25.20 & 33.80 & 8.60 & 3.27 & 3.49 \\
\hline & Mean & 25.50 & 33.80 & 8.30 & 3.33 & 3.93 \\
\hline & SD & 0.40 & 0.13 & 0.31 & 0.05 & 0.71 \\
\hline & $\mathrm{CV}$ & 0.02 & 0.01 & 0.04 & 0.02 & 0.18 \\
\hline \multirow[t]{7}{*}{$15-25$} & 25 & 18.60 & 22.90 & 4.30 & 3.51 & 4.37 \\
\hline & 20 & 18.30 & 22.40 & 4.10 & 3.47 & 4.47 \\
\hline & 15 & 18.50 & 22.90 & 4.40 & 3.30 & 3.37 \\
\hline & 10 & 18.80 & 22.80 & 4.00 & 3.19 & 3.60 \\
\hline & Mean & 18.60 & 22.80 & 4.20 & 3.37 & 3.95 \\
\hline & SD & 0.21 & 0.24 & 0.18 & 0.15 & 0.55 \\
\hline & $\mathrm{CV}$ & 0.01 & 0.01 & 0.04 & 0.04 & 0.14 \\
\hline
\end{tabular}

$\mathrm{SD}=$ Standard Deviation; $\mathrm{CV}=$ Coefficient of Variation; $\mathrm{T}_{\max }=$ Maximum temperature; $\mathrm{T}_{\min }=$ Minimum temperature; $\mathrm{A}=\mathrm{Amplitude} ; \mathrm{L}_{\min }=$ Lag at minimum temperature; $\mathrm{L}_{\max }=\mathrm{Lag}$ at maximum temperature

Table 3: Total solids

\begin{tabular}{|c|c|c|c|c|c|c|c|c|c|c|}
\hline \multirow{3}{*}{$\begin{array}{l}\text { Temperature } \\
\text { Cycle } \\
\left({ }^{\circ} \mathrm{C}\right)\end{array}$} & \multirow[b]{3}{*}{$\begin{array}{l}\text { HRT } \\
\text { (d) }\end{array}$} & \multicolumn{3}{|c|}{ TS } & \multicolumn{3}{|c|}{ TFS } & \multicolumn{3}{|c|}{ TVS } \\
\hline & & \multirow[b]{2}{*}{$\begin{array}{l}\text { Mean } \\
(\mathrm{g} / \mathrm{L})\end{array}$} & \multicolumn{2}{|c|}{ Reduction } & \multirow[b]{2}{*}{$\begin{array}{l}\text { Mean } \\
(\mathrm{g} / \mathrm{L})\end{array}$} & \multicolumn{2}{|c|}{ Reduction } & \multirow[b]{2}{*}{$\begin{array}{l}\text { Mean } \\
(\mathrm{g} / \mathrm{L})\end{array}$} & \multicolumn{2}{|c|}{ Reduction } \\
\hline & & & $(\mathrm{g} / \mathrm{L})$ & $(\%)$ & & (g/L) & $(\%)$ & & $(\mathrm{g} / \mathrm{L})$ & $(\%)$ \\
\hline \multirow[t]{4}{*}{$20-40$} & 25 & 40.60 & 23.65 & 36.8 & 10.60 & 3.39 & 24.2 & 30.0 & 20.26 & 40.3 \\
\hline & 20 & 41.30 & 22.95 & 35.7 & 10.90 & 3.09 & 26.4 & 30.4 & 19.86 & 39.5 \\
\hline & 15 & 44.10 & 20.45 & 31.4 & 11.80 & 2.19 & 15.7 & 32.3 & 17.96 & 35.7 \\
\hline & 10 & 44.80 & 19.45 & 30.3 & 12.10 & 1.89 & 13.5 & 32.7 & 17.56 & 34.9 \\
\hline \multirow[t]{4}{*}{$15-25$} & 25 & 39.70 & 24.55 & 38.2 & 9.40 & 4.59 & 32.8 & 30.3 & 19.96 & 39.7 \\
\hline & 20 & 44.90 & 19.35 & 30.1 & 10.20 & 3.79 & 27.1 & 34.7 & 15.56 & 30.9 \\
\hline & 15 & 47.30 & 16.95 & 26.4 & 10.60 & 3.39 & 25.7 & 36.7 & 13.56 & 26.9 \\
\hline & 10 & 48.60 & 15.65 & 24.4 & 11.00 & 2.99 & 21.4 & 37.4 & 12.66 & 25.2 \\
\hline
\end{tabular}

TS = Total Solids; TFS = Total Fixed Solids; TVS = Total Volatile Solids; Initial TS = 64.25 $\mathrm{g} \mathrm{L}^{-1}$; Initial VS = 50.26 g L ${ }^{-1}$; Initial FS = 13.99 $\mathrm{g} \mathrm{L}^{-1}$

Table 4: Suspended solids

\begin{tabular}{|c|c|c|c|c|c|c|c|c|c|c|}
\hline \multirow{3}{*}{$\begin{array}{l}\text { Temperature } \\
\text { Cycle } \\
\left({ }^{\circ} \mathrm{C}\right) \\
\end{array}$} & \multirow[b]{3}{*}{$\begin{array}{l}\text { HRT } \\
\text { (d) }\end{array}$} & \multicolumn{3}{|c|}{ TSS } & \multicolumn{3}{|c|}{ FSS } & \multicolumn{3}{|c|}{ VSS } \\
\hline & & \multirow[b]{2}{*}{$\begin{array}{l}\text { Mean } \\
(\mathrm{g} / \mathrm{L})\end{array}$} & \multicolumn{2}{|c|}{ Reduction } & \multirow[b]{2}{*}{$\begin{array}{l}\text { Mean } \\
(\mathrm{g} / \mathrm{L})\end{array}$} & \multicolumn{2}{|c|}{ Reduction } & \multirow{2}{*}{$\begin{array}{l}\text { Mean } \\
(\mathrm{g} / \mathrm{L})\end{array}$} & \multicolumn{2}{|c|}{ Reduction } \\
\hline & & & $(\mathrm{g} / \mathrm{L})$ & $(\%)$ & & $(\mathrm{g} / \mathrm{L})$ & $(\%)$ & & $(\mathrm{g} / \mathrm{L})$ & $(\%)$ \\
\hline \multirow[t]{4}{*}{$20-40$} & 25 & 35.40 & 6.85 & 16.21 & 9.40 & 1.85 & 16.44 & 26.00 & 5.00 & 16.13 \\
\hline & 20 & 37.20 & 5.05 & 11.95 & 4.40 & 6.85 & 60.89 & 32.80 & -1.80 & -5.80 \\
\hline & 15 & 39.80 & 2.45 & 5.80 & 5.00 & 6.25 & 55.56 & 34.80 & -3.80 & -12.26 \\
\hline & 10 & 42.30 & -0.05 & -0.11 & 7.00 & 4.25 & 37.78 & 35.30 & -4.30 & -13.87 \\
\hline \multirow[t]{4}{*}{$15-25$} & 25 & 35.40 & 6.85 & 16.21 & 9.40 & 1.85 & 16.44 & 26.00 & 5.00 & 16.13 \\
\hline & 20 & 39.50 & 2.75 & 6.51 & 10.60 & 0.65 & 5.78 & 28.90 & 2.10 & 6.77 \\
\hline & 15 & 42.30 & -0.05 & -0.11 & 10.70 & 0.55 & 4.89 & 31.60 & -0.70 & -1.94 \\
\hline & 10 & 46.00 & -3.75 & -8.88 & 10.90 & 0.35 & 3.11 & 35.10 & -4.10 & -13.23 \\
\hline
\end{tabular}

TSS = Total Suspended Solids; FSS = Fixed Suspended Solids; VSS = Volatile Suspended Solids; Initial TTS = $42.25 \mathrm{~g} \mathrm{~L}^{-1}$; Initial VSS = 31.00 $\mathrm{g} \mathrm{L}^{-1}$; Initial FSS $=11.25 \mathrm{~g} \mathrm{~L}^{-1}$

Table 5: Total kjeldahl and ammonium nitrogen

\begin{tabular}{|c|c|c|c|c|c|c|c|c|}
\hline \multirow{3}{*}{$\begin{array}{l}\text { Temperature } \\
\text { Cycle } \\
\left({ }^{\circ} \mathrm{C}\right)\end{array}$} & \multirow{3}{*}{$\begin{array}{l}\text { HRT } \\
\text { (d) }\end{array}$} & \multicolumn{3}{|c|}{ TKN } & \multicolumn{3}{|c|}{$\mathrm{NH}_{4}-\mathrm{N}$} & \multirow[b]{3}{*}{$\frac{\mathrm{NH}_{4}-\mathrm{N}}{\mathrm{TKN}}$} \\
\hline & & \multirow{2}{*}{$\begin{array}{l}\text { Mean } \\
(\mathrm{g} / \mathrm{L})\end{array}$} & \multicolumn{2}{|c|}{ Reduction } & \multirow{2}{*}{$\begin{array}{l}\text { Mean } \\
(\mathrm{g} / \mathrm{L})\end{array}$} & \multicolumn{2}{|c|}{ Reduction } & \\
\hline & & & $(\mathrm{g} / \mathrm{L})$ & $(\%)$ & & $(\mathrm{g} / \mathrm{L})$ & $(\%)$ & \\
\hline $20-40$ & $\begin{array}{l}25 \\
20 \\
15 \\
10 \\
\end{array}$ & $\begin{array}{l}1.87 \\
2.04 \\
2.24 \\
2.37 \\
\end{array}$ & $\begin{array}{l}1.93 \\
1.76 \\
1.56 \\
1.43 \\
\end{array}$ & $\begin{array}{l}51.32 \\
46.32 \\
41.05 \\
37.63 \\
\end{array}$ & $\begin{array}{l}3.06 \\
3.43 \\
3.61 \\
3.70 \\
\end{array}$ & $\begin{array}{l}-0.61 \\
-0.98 \\
-1.16 \\
-1.25 \\
\end{array}$ & $\begin{array}{l}-40.00 \\
-40.06 \\
-47.35 \\
-51.02 \\
\end{array}$ & $\begin{array}{l}1.63 \\
1.68 \\
1.61 \\
1.56 \\
\end{array}$ \\
\hline $15-25$ & $\begin{array}{l}25 \\
20 \\
15 \\
10\end{array}$ & $\begin{array}{l}1.78 \\
2.02 \\
2.12 \\
2.30 \\
\end{array}$ & $\begin{array}{l}2.02 \\
1.78 \\
1.68 \\
1.50 \\
\end{array}$ & $\begin{array}{l}53.16 \\
46.84 \\
44.21 \\
39.47 \\
\end{array}$ & $\begin{array}{l}3.03 \\
3.21 \\
3.24 \\
3.45 \\
\end{array}$ & $\begin{array}{l}-0.68 \\
-0.76 \\
-0.79 \\
-1.00 \\
\end{array}$ & $\begin{array}{l}-23.67 \\
-31.02 \\
-32.45 \\
-40.82 \\
\end{array}$ & $\begin{array}{l}1.85 \\
1.59 \\
1.52 \\
1.50\end{array}$ \\
\hline
\end{tabular}

$\mathrm{TKN}=$ Total Kjeldahl Nitrogen; $\mathrm{NH}_{4}-\mathrm{N}=$ Ammonium Nitrogen; Initial TKN $=3.80 \mathrm{~g} \mathrm{~L}^{-1}$; Initial NH $4-\mathrm{N}=2.45 \mathrm{~g} \mathrm{~L}{ }^{-1} ; \mathrm{Initial} \mathrm{NH}_{4}-\mathrm{N} / \mathrm{TKN}=0.64$ 
Am. J. Biochem. \& Biotech., 7 (4): 146-162, 2011

Table 6: Volatile fatty acids

\begin{tabular}{|c|c|c|c|c|c|c|c|c|c|c|c|}
\hline \multirow{2}{*}{$\begin{array}{l}\text { Temp } \\
\text { Cycle } \\
\left({ }^{\circ} \mathrm{C}\right)\end{array}$} & \multirow[b]{2}{*}{$\begin{array}{l}\text { HRT } \\
\text { (d) }\end{array}$} & \multicolumn{10}{|c|}{ Concentration (mg/L) } \\
\hline & & $\begin{array}{l}\text { Acetic } \\
\text { acid }\end{array}$ & $\begin{array}{l}\text { Propionic } \\
\text { acid }\end{array}$ & $\begin{array}{l}\text { i-Butyric } \\
\text { acid }\end{array}$ & $\begin{array}{l}\text { n-Butyric } \\
\text { acid }\end{array}$ & $\begin{array}{l}\text { i-Valeric } \\
\text { acid }\end{array}$ & $\begin{array}{l}n-\text { Valeric } \\
\text { acid }\end{array}$ & $\begin{array}{l}\text { i-Caproic } \\
\text { acid }\end{array}$ & $\begin{array}{l}\text { n-Caproic } \\
\text { acid }\end{array}$ & $\begin{array}{l}\text { Heptanoic } \\
\text { acid }\end{array}$ & $\begin{array}{l}\text { Total as } \\
\text { acetic acid }\end{array}$ \\
\hline \multirow[t]{4}{*}{$20-40$} & 25 & 5.5 & 26.60 & 15.60 & 8.40 & 9.80 & $<0.01$ & 11.70 & 3.90 & 5.70 & 60.0 \\
\hline & 20 & 44.0 & $<0.01$ & $<0.01$ & $<0.01$ & $<0.01$ & $<0.01$ & $<0.01$ & $<0.01$ & $<0.01$ & 44.7 \\
\hline & 15 & 3.0 & 26.00 & 10.70 & 11.30 & 9.70 & 1.30 & 3.30 & 2.40 & 2.50 & 50.0 \\
\hline & 10 & 53.8 & 56.90 & 35.70 & 8.20 & 11.50 & 5.00 & 9.00 & 4.70 & 16.90 & 154.8 \\
\hline \multirow[t]{4}{*}{$15-25$} & 25 & 54.1 & 73.60 & 13.70 & 18.30 & 3.90 & $<0.01$ & 2.20 & 5.40 & 20.00 & 151.0 \\
\hline & 20 & 70.8 & 102.10 & 19.10 & 26.00 & 19.80 & 4.70 & 6.10 & 6.10 & 58.40 & 231.8 \\
\hline & 15 & 207.4 & 92.00 & 18.50 & 27.60 & 19.70 & 4.10 & 6.60 & 4.30 & 59.40 & 362.5 \\
\hline & 10 & 645.0 & 431.40 & 139.70 & 38.60 & 36.00 & 25.00 & 22.70 & 17.80 & 30.10 & 1187.0 \\
\hline \multicolumn{2}{|c|}{ Raw manure } & 1548.4 & 283.50 & 44.50 & 60.50 & 40.20 & 21.00 & 7.00 & 11.30 & 37.10 & 1913.0 \\
\hline
\end{tabular}

Table 7: Biogas composition*

\begin{tabular}{|c|c|c|c|c|c|c|}
\hline \multirow{2}{*}{$\begin{array}{l}\text { Temperature } \\
\text { Cycle } \\
\left({ }^{\circ} \mathrm{C}\right)\end{array}$} & \multirow{2}{*}{$\begin{array}{l}\text { HRT } \\
\text { (d) }\end{array}$} & \multicolumn{5}{|c|}{ Gas composition } \\
\hline & & $\mathrm{CH}_{4}(\%)$ & $\mathrm{SD}(\%)$ & $\mathrm{CO}_{2}(\%)$ & $\mathrm{SD}(\%)$ & Others** $(\%)$ \\
\hline \multirow[t]{4}{*}{$20-40$} & 25 & 69.0 & 1.73 & 25.8 & 1.490 & 5.2 \\
\hline & 20 & 67.6 & 1.31 & 28.6 & 2.254 & 3.8 \\
\hline & 15 & 62.6 & 0.98 & 37.3 & 0.980 & 0.1 \\
\hline & 10 & 64.9 & 0.98 & 34.9 & 0.990 & 0.2 \\
\hline \multirow[t]{4}{*}{$15-25$} & 25 & 71.8 & 2.13 & 25.3 & 0.970 & 3.0 \\
\hline & 20 & 69.6 & 1.79 & 27.3 & 0.900 & 3.1 \\
\hline & 15 & 47.8 & 0.58 & 44.0 & 1.280 & 8.2 \\
\hline & 10 & 44.6 & 0.53 & 43.6 & 1.840 & 11.8 \\
\hline
\end{tabular}

* Each mean represents the average of 12 samples; **: Nitrogen, hydrogen sulphide, carbon monoxide; SD = Standard Deviation

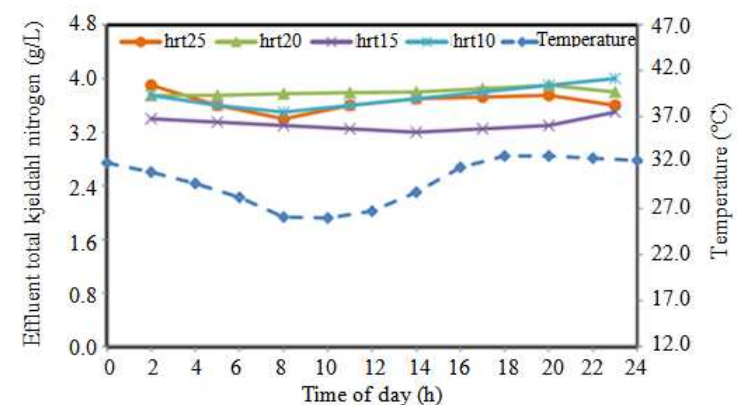

(a) $20-40^{\circ} \mathrm{C}$ temperature cycle

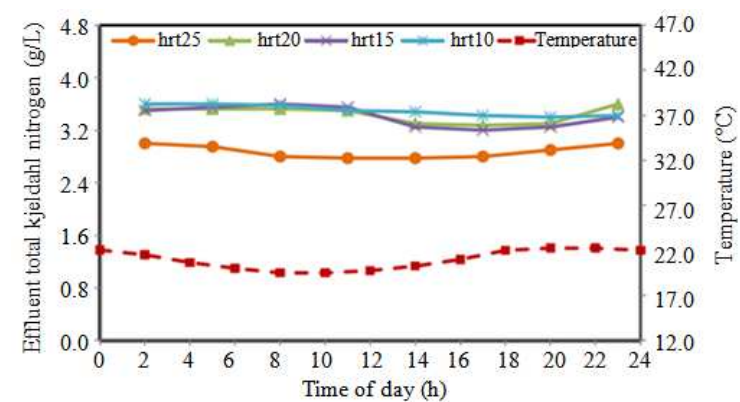

(b) $15-25^{\circ} \mathrm{C}$ temperature cycle

Fig. 9: Diurnal variations in total kjeldahl nitrogen

Biogas production and composition: Figure 10 shows the daily biogas production under various operating conditions. The diurnal variations in the mean rate of biogas production for the $25 \mathrm{~h} \mathrm{HRT}$ are shown in Fig. 11 .

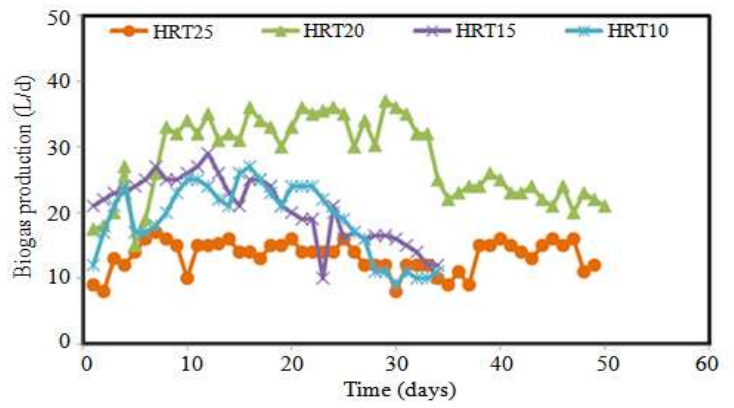

(a) $20-40^{\circ} \mathrm{C}$ temperature cycle

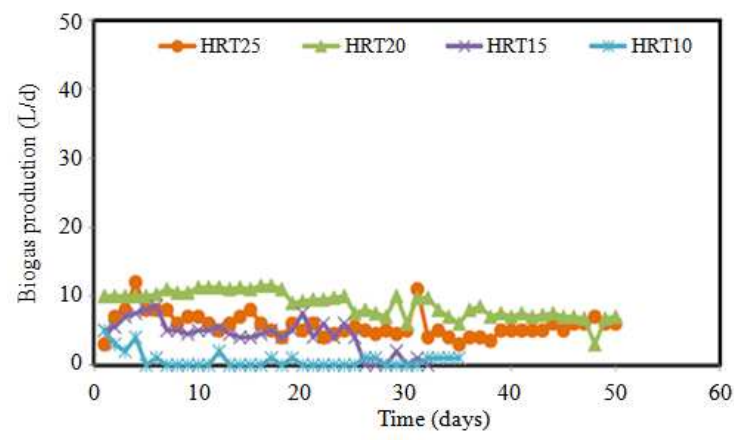

(b) $15-25^{\circ} \mathrm{C}$ temperature cycle

Fig. 10: Daily biogas production

A summary of the average composition of the biogas collected under the various treatment combinations is shown in Table 7. 


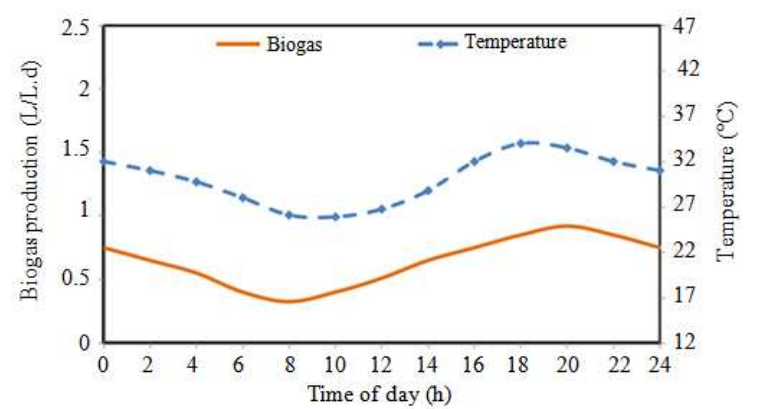

(a) $20-40^{\circ} \mathrm{C}$ temperature cycle

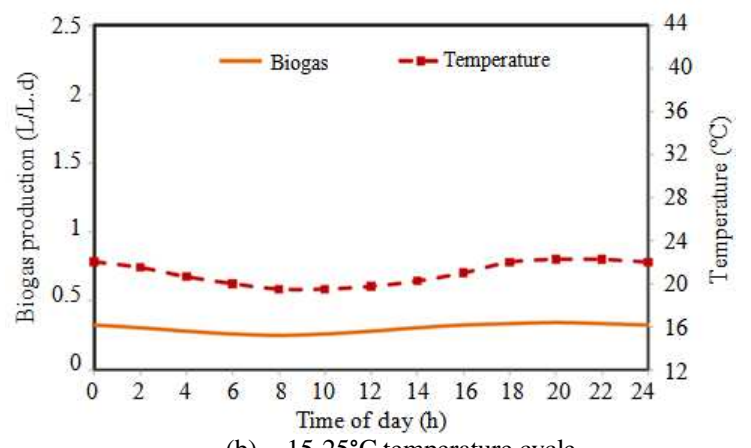

(b) $15-25^{\circ} \mathrm{C}$ temperature cycle

Fig. 11: Diurnal variations in biogas production at the 25 d HRT

\section{DISCUSSION}

Temperature: The mean minimum and maximum temperatures for the reactors operating under the 20$40^{\circ} \mathrm{C}$ temperature cycle were 25.5 and $33.8^{\circ} \mathrm{C}$, respectively giving a mean amplitude of $\pm 8.3^{\circ} \mathrm{C}$. The mean minimum and maximum temperatures for the 15 $25^{\circ} \mathrm{C}$ temperature cycle were 18.6 and $22.8^{\circ} \mathrm{C}$, respectively giving a mean amplitude of $\pm 4.2^{\circ} \mathrm{C}$. Relative to chamber temperature, there was a lag in the reactor temperature at both the maximum and minimum temperatures of both temperature cycles. The mean lag at the maximum and minimum temperatures for the 15 $25^{\circ} \mathrm{C}$ temperature cycle and the $20-40{ }^{\circ} \mathrm{C}$ temperature cycle were $3.37 \mathrm{~h}$ and $3.95 \mathrm{~h}$ and $3.38 \mathrm{~h}$ and $4.35 \mathrm{~h}$, respectively. This was due to the significant differences between the density of air in the chamber and the liquid medium in the reactor and the thermal properties of the reactors' walls which affected the rate of heat transfer to and from the reactors.

Total solids: Diurnally cyclic variations in the effluent total solids were observed under the $20-40^{\circ} \mathrm{C}$ temperature cycle. However, the diurnally cyclic variations in total solids were not distinct at the 15 and
$10 \mathrm{~d}$ HRTs under the $15-25^{\circ} \mathrm{C}$ temperature cycle. Similar diurnally cyclic variations were observed for the effluent total volatile solids and total fixed solids.

For the $20-40^{\circ} \mathrm{C}$ temperature cycle, the TS reductions ranged from 30.3-36.8\% (19.45-23.65 $\left.\mathrm{g} \mathrm{L}^{-1}\right)$, the TVS reductions ranged from $34.9-40.3 \%$ (17.56$20.26 \mathrm{~g} \mathrm{~L}^{-1}$ ) while the TFS reductions ranged from $13.5-24.2 \%$ (1.89-3.39 $\left.\mathrm{g} \mathrm{L} \mathrm{L}^{-1}\right)$, depending on the hydraulic retention time. For the $15-25^{\circ} \mathrm{C}$ temperature cycle, the TS reductions ranged from 24.4-38.3\% (15.65-24.55 $\left.\mathrm{g} \mathrm{L}^{-1}\right)$, the TVS reductions ranged from $25.2-39.7 \% \quad\left(12.66-19.96 \mathrm{~g} \mathrm{~L} \mathrm{~L}^{-1}\right)$ while the TFS reductions ranged from $21.4-32.8 \%\left(2.99-4.59 \mathrm{~g} \mathrm{~L}^{-1}\right)$, depending on the hydraulic retention time.

Higher TS reductions were achieved with the high diurnal temperature cycle $\left(20-40^{\circ} \mathrm{C}\right)$ than those with the lower diurnal temperature cycle $\left(15-25^{\circ} \mathrm{C}\right)$ at all HRTs. Walkie et al. (2004) obtained the highest TS reduction of $46.7 \%$ during the treatment of dairy manure at a low temperature range of $11.7-32.4^{\circ} \mathrm{C}$. Elango et al. (2007) achieved a TS reduction of $87.6 \%$ during the treatment of solid wastes over the temperature range of $26-36^{\circ} \mathrm{C}$. The TS reduction achieved in the present study (25.2$39.7 \%$ ) are within the values reported in the literature.

The hydraulic retention time (HRT) had a significant effect on the TS reduction in the present study. Generally, the shorter the HRT, the lower the solids reductions. Ribas and Barana (2003) reported that a decrease in the HRT from 16.6-9.7 $\mathrm{d}$ increased the TS of anaerobically treated wastewater from 2.3$4.8 \%$. Umana et al. (2008) reported an increase in the TS removal efficiency from 14.6-68.8\% when the HRT was increased from 1-5.5 d during the treatment of dairy manure. Rico et al. (2011a) noted that the concentration of TS increased from $39.2-45.3 \mathrm{~g} \mathrm{~L}^{-1}$ as the HRT was decreased from 20-10 d during the treatment of dairy manure.

The absolute values of TVS reductions $(\mathrm{g} / \mathrm{L})$ were generally lower than those of the TS. This may be explained by the fact that volatile solids are converted to microbial biomass, volatile fatty acids (VFAs) and biogas. While microbial biomass contributes to the total solids content of the effluent, the VFAs are evaporated at $105^{\circ} \mathrm{C}$ and therefore, are not detected in TS measurements (Ghaly and Echiegu, 1993).

The HRT had a significant effect on the effluent TVS. Generally, the shorter the HRT the lower the TVS reduction. De la Rubia et al. (2002) reported an increase in the TVS reduction (from 53-73\%) as the HRT increased from 27-75 d. Ribas and Barana (2003) reported an increase in the TVS from $1.6-4.3 \%$ in a wastewater treatment system as the HRT was decreased from 16.6-9.7 d. Umana et al. (2008) reported an 
increase in the TVS reduction (from 26.3-78.8\%) during the treatment of dairy manure as the HRT was increased from 1-5.5 d. Rico et al. (2011b) noted that the concentration of TVS increased from 23.7-27.8 g $\mathrm{L}^{-1}$ during anaerobic treatment of dairy manure as the HRT decreased from 20-10 d. The reduction in the TVS obtained in this study (25.2-39.7\%) were within the range reported in the literature.

The diurnal cycling temperature also affected the TVS reductions, the higher the temperature the higher the TVS reduction. Walkie et al. (2004) achieved the highest TVS reduction of $60.4 \%$ at a temperature of $32.4^{\circ} \mathrm{C}$ during the treatment of dairy manure wastewaters. Elango et al. (2007) reported $88.1 \%$ reduction in TVS over the temperature range of 26$36^{\circ} \mathrm{C}$. Trazcinski and Stuckey (2011) reported TVS reductions in the range of $40-75 \%$ at the temperatures of $21-35^{\circ} \mathrm{C}$ during anaerobic digestion of solid waste. The TVS reductions achieved in this study (25.2-39.7 $\%)$ are within the range reported in the literature.

Fixed solids represent the inorganic components of the waste and it consists of potassium, sodium, calcium, magnesium, iron, copper and other minerals (Ghaly and Echiegu, 1993). Unlike carbon and nitrogen, little or no significant losses in these inorganic compounds are generally expected to occur since they do not play a very significant role in the anaerobic digestion process. In this system, a fixed solid recovery ranging from 64.3-112.0\% was recorded. Converse et al. (1977) reported a fixed solid recovery ranging from $90-104 \%$. In this study, the losses in fixed solids may have been due to inadequate mixing of the bioreactor contents which may have resulted in the settling out of the inorganic mineral resulting in the lower concentration of these compounds in the effluent. They may, also, have been due to the fact that changes in the nature of these inorganic compounds may have occurred at the higher temperature $\left(550^{\circ} \mathrm{C}\right)$ used in the determination of total ash content.

Suspended solids: Diurnally cyclic variations in the TSS were observed at all hydraulic retention times for both operating temperature cycles. The variations were in phase with the measured bioreactor temperature at all HRTs under the $20-40^{\circ} \mathrm{C}$ diurnal temperature cycle. They were, also, in phase at the $25 \mathrm{~d}$ HRT under the 15$25^{\circ} \mathrm{C}$ diurnal temperature cycle but out of phase with the reactor temperature by $7-11 \mathrm{~h}$ for other HRTs (10$20 \mathrm{~d})$. The variation in Volatile Suspended Solids (VSS) followed a pattern similar to that of TSS, but the fixed suspended solids did not exhibit any diurnally cyclic variation at all HRTs.
With the $20-40{ }^{\circ} \mathrm{C}$ diurnal temperature cycle, the TSS reductions ranged from $-0.11-16.21 \%(-0.05-6.85$ $\mathrm{g} \mathrm{L}^{-1}$ ), the FSS reductions ranged from 16.44-60.89\% (1.85-6.85 $\left.\mathrm{g} \mathrm{L}^{-1}\right)$ while the VSS reductions ranged from $-13.87-16.13 \%\left(-4.30-5.00 \mathrm{~g} \mathrm{~L}^{-1}\right)$, depending on the HRT. With the $15-25^{\circ} \mathrm{C}$ diurnal temperature cycle, the TSS reductions ranged from $-8.88-16.21 \%(-3.75-6.85$ $\left.\mathrm{g} \mathrm{L}^{-1}\right)$, the FSS reductions ranged from $3.11-16.44 \%$ (0.35-1.85 $\left.\mathrm{g} \mathrm{L}^{-1}\right)$ while the VSS ranged from -13.23$16.13 \%$ (-4.10-5.00 $\left.\mathrm{g} \mathrm{L}^{-1}\right)$, depending on HRT.

The TSS reductions achieved in this study ranged from $-8.88-16.21 \%$, the longer the HRT the higher the TSS reductions. Kayranli and Ugurlu (2011) found that the TSS concentrations in the anaerobic treatment of wastewater is higher at shorter HRTs. Lew et al. (2004) noted an increase in TSS reduction during the treatment of domestic wastewater as the HRT was increased from 3-24 h. Alvarez et al. (2008) reported TSS reductions of 63 and $85 \%$ during the anaerobic digestion of wetland plants at HRTs of 5 and $16 \mathrm{~h}$, respectively.

The results also showed that the diurnal cycling temperature had a significant effect on the TSS reductions. Higher TSS reductions were observed with the higher diurnal temperature cycle. Lew et al. (2004) reported increased TSS concentrations (from 17-120 $\mathrm{mg} \mathrm{\textrm {L } ^ { - 1 }}$ ) as the temperature decreased from $28-10^{\circ} \mathrm{C}$. Kayranli and Ugurlu (2011) found that as the temperature was decreased from $25-10^{\circ} \mathrm{C}$ during anaerobic treatment of wastewater the concentration of TSS increased from 6-10 $\mathrm{mg} \mathrm{L}^{-1}$.

The volatile suspended solids are usually used as an indicator of the cell mass concentration of the system. Higher loading rates (shorter HRTs) promote the formation of larger populations of anaerobic bacteria. The formation of bacteria contributes to the volatile suspended solids content of the system as is reflected in the higher effluent suspended solids content (lower suspended solids reductions) recorded at shorter hydraulic retention times. If the mixing process is not completely perfect, longer hydraulic retention times allows the settling out of some of the settleable components of the suspended solids, the formation of scum at the liquid surface and the attachment of biomass to the walls of the bioreactor. These phenomena would prevent the suspended solids from being washed out with the effluent.

The results showed that higher VSS reductions were observed with longer HRTs. Maharaj and Elefsiniotis (2001) reported VSS reduction of 71.8, 72.9 and $74.6 \%$ during the anaerobic digestion of industrial and municipal wastewaters at HRT of 30,48 and $60 \mathrm{~h}$, 
respectively. Chu et al. (2011) noted VSS concentrations of 21.50 and $13.25 \mathrm{~g} \mathrm{~L}^{-1}$ during molasses fermentation at HRTs of 4 and $8 \mathrm{~h}$, respectively. AlGhusain et al. (2002) reported VSS concentrations of 4725 and $4614 \mathrm{mg} \mathrm{L}^{-1}$ during anaerobic sludge digestion at HRTs of 10-20 d, respectively.

The results also showed that the VSS reductions were significantly affected by the reaction temperature. Higher VSS reductions were obtained with the high diurnal temperature cycle. Maharaj and Elefsiniotis (2001) found that the VSS reduction increased from 50$74.6 \%$ during the anaerobic digestion of industrial and municipal wastewaters as the temperature was increased from $8-25^{\circ} \mathrm{C}$. Al-Ghusain et al. (2002) reported VSS concentrations of 4725 and $4549 \mathrm{mg} \mathrm{L}^{-1}$ during anaerobic sludge digestion at the temperatures of 30 and $40^{\circ} \mathrm{C}$, respectively.

Nitrogen: Fresh undigested dairy manure contains a substantial amount of organic nitrogen. During the anaerobic digestion process, some of the organic nitrogen is converted by microbial action into ammonium nitrogen. The oxidized forms of Nitrogen $\left(\mathrm{NO}_{2}\right.$ and $\left.\mathrm{NO}_{3}\right)$ cannot exist in anaerobic environments, hence, the forms of nitrogen that can be present is limited to organic and ammonium nitrogen. These two forms are customarily combined and referred to as Total Kjeldahl Nitrogen (TKN).

The effluent $\mathrm{NH}_{4}-\mathrm{N}$ concentration showed diurnally cyclic variations at the 25 and 20 d HRTs under the $20-40^{\circ} \mathrm{C}$ diurnal temperature cycle and at all HRTs under the $15-25^{\circ} \mathrm{C}$ diurnal temperature cycle. The TKN and $\mathrm{NH}_{4}-\mathrm{N}$ concentrations increased with decreases in the HRT under both diurnal temperature cycles. TKN reductions ranged from $2.7-20.31 \%$ while $\mathrm{NH}_{4}-\mathrm{N}$ reductions ranged from $3.6-27.6 \%$, depending on operating conditions.

The higher reductions in the nitrogen compounds achieved at the longer HRT were probably because the hydrolytic bacteria responsible for the conversion of organic nitrogen to $\mathrm{NH}_{4}-\mathrm{N}$ were offered greater opportunity to effect the hydrolytic process. Diurnal fluctuations were less with the $15-25^{\circ} \mathrm{C}$ diurnal temperature cycle compared to the $20-40^{\circ} \mathrm{C}$ diurnal temperature cycle. The effects of a diurnally cyclic temperature may, therefore, have been less felt at the $15-25^{\circ} \mathrm{C}$ temperature cycle by the hydrolytic bacteria. This may have led to a higher conversion rate of organic nitrogen to ammonium nitrogen and, hence, to higher losses. The ratio of ammonium to total kjeldahl nitrogen in the effluent sample ranged from 1.52-1.85. These values are comparable to those reported by other researchers (Converse et al., 1977; Rico et al., 2011a;
Sooknah et al., 2004). The aim of anaerobic treatment is to promote the conversion of organic nitrogen into ammonium nitrogen.

Volatile fatty acids: The VFAs concentration in the effluent ranged from $45-1200 \mathrm{mg} \mathrm{L}^{-1}$, depending on the operating conditions. The effluent VFAs concentration generally increased with decreases in the HRT and higher VFAs concentrations were recorded at the lower temperature cycle. At the $20-40^{\circ} \mathrm{C}$ diurnal temperature cycle, the VFAs (measured as acetic acid) first decreased from $60-44.7 \mathrm{mg} \mathrm{L}^{-1}$ as the HRT was reduced from 25-20 d. Further reductions in the HRT resulted in increasingly higher concentrations of VFAs, up to $154.8 \mathrm{mg} \mathrm{L}^{-1}$ was recorded at the 10 day HRT. Among the individual volatile acids, propionic acid had the highest concentration at all but $20 \mathrm{~d}$ HRT. At the 20 d HRT, only acetic acid was detected in measurable levels. At the $15-25^{\circ} \mathrm{C}$ diurnal temperature cycle, the total volatile acids concentration increased from 151.0

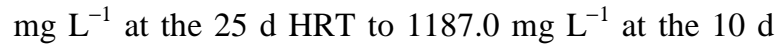
HRT. Propionic acid concentration was higher than that of the rest at the 25 and $20 \mathrm{~d}$ HRTs while acetic acid concentration was the highest at the 15 and $10 \mathrm{~d}$ HRTs.

The HRT has a significant influence on the production of VFAs, the shorter the HRT the higher the VFAs concentration. Colmenarejo et al. (2004) reported that the highest VFAs production was achieved when the HRT was decreased from 5.2-1.7 h. Lew et al. (2004) reported that the VFA concentration increased when the HRT was decreased from 24-3 h during the treatment of domestic wastewater. Elefkiniotis and Oldham (1994) reported an increase in VFA production when the HRT was increased from 6-12 $\mathrm{h}$ which then decreased with further increase in the HRT to $15 \mathrm{~h}$. Maharaj and Elefsiniotis (2001) noted highest VFA concentration at an HRT of $30 \mathrm{~h}$ as appose to 48 and 60 $\mathrm{h}$ while an aerobically treating wastewaters. De la Rubia et al. (2002) found that the VFA production increased from 5532-6432 $\mathrm{mg} \mathrm{L}^{-1}$ as the HRT decreased from 75-20 days.

The results also showed the importance of temperature on the production of VFAs. Higher VFA production was observed with lower diurnal cyclic temperature. Maharaj and Elefsiniotis (2001) noted increased VFA concentrations as the temperature was increased from $8-25^{\circ} \mathrm{C}$, which then decreased upon further increase in temperature (from $25-35^{\circ} \mathrm{C}$ ). Choorit and Wisarnwan (2007) noted that the VFA concentration slightly increased from 160.71-166.64 $\mathrm{mg} \mathrm{L}^{-1}$ as the temperature decreased from $43-37^{\circ} \mathrm{C}$ and from 245.07-338.71 mg $\mathrm{L}^{-1}$ as the temperature decreased from $55-49^{\circ} \mathrm{C}$. De la Rubia et al. (2002) 
observed an increase in VFA production from 830-5688 $\mathrm{mg} \mathrm{L}^{-1}$ during digestion of anaerobic municipal sludge as the temperature increased from $35-55^{\circ} \mathrm{C}$.

However, values of effluent VFAs concentrations reported in the literature vary depending on the type of waste. Converse et al. (1977) reported effluent VFAs concentrations of $423-1113 \mathrm{mg} \mathrm{L}^{-1}$ (as acetic acid) using CSTR operated on dairy manure of $12.8-13.4 \%$ TS content and a temperature of $35^{\circ} \mathrm{C}$. Jeyanayagam and Collins (1984) reported effluent VFAs concentrations of $754-1515 \mathrm{mg} \mathrm{L}^{-1}$ using laboratory digesters operated on dairy manure of $4.0-6.5 \%$ TS content at hydraulic retention times of 15-20 d and a temperature of $35^{\circ} \mathrm{C}$. Lo and Liao (1986) reported a VFAs concentration of $584 \mathrm{mg} \mathrm{L}^{-1}$ using a fixed film reactor operated on a screened dairy manure of $3.7 \%$ TS content at an HRT of $20 \mathrm{~d}$ and a temperature of $12^{\circ} \mathrm{C}$. Peck et al. (1986) reported effluent VFAs concentrations of $64-98 \mathrm{mg} \mathrm{L}^{-1}$ using manure of 3458$4109 \mathrm{mg} \mathrm{L}^{-1} \mathrm{TS}$ at 10-25 d HRTs and a temperature of $35^{\circ} \mathrm{C}$. The VFA concentrations observed in this study are within the range of values reported in the literature.

Organic wastes are first converted to long chain fatty acids in the acidogenic stage of anaerobic digestion processes. Volatile acids are further converted to acetic acid which together with hydrogen and formate serve as substrates for methane bacteria. Different species of anaerobic bacteria are involved in the conversion of specific long chain fatty acids to acetic acid. The increased proportion of propionic acid relative to acetic acid indicated that the different species of anaerobic bacteria did not respond in a similar manner to the effect of diurnally cyclic temperature. The fact that individual VFAs are removed at different rates under adverse conditions such as a drop in temperature has been observed by other researchers (Peck et al., 1986). A lower methane yield was recorded under the conditions in which the propionic acid concentration was high indicating that propionic acid was not available for conversion to methane.

Biogas production: There appears to be a definite relationship between the bioreactor temperature and the rate of biogas production. Biogas production followed a diurnally cyclic pattern similar to that of the bioreactor temperature under most operating conditions. The highest reactor temperature and the maximum rate of biogas production occurred at the $18.00 \mathrm{~h}$ while the lowest reactor temperature and the minimum biogas production rate occurred at the $09.00 \mathrm{~h}$. This trend was observed at all HRTs when operating under the 20$40^{\circ} \mathrm{C}$ diurnal temperature cycle and at the $20 \mathrm{~d}$ HRT when operated under the $15-25^{\circ} \mathrm{C}$ diurnal temperature cycle. The daily amplitude of the gas production cycle was higher under the $20-40^{\circ} \mathrm{C}$ diurnal temperature cycle than that observed under $15-25^{\circ} \mathrm{C}$ diurnal temperature cycle. Smaller amplitude in the gas production cycle was recorded in the lower operating temperature cycle because of the smaller diurnal amplitude of the reactor temperature. With digesters operated under stressful conditions such as a combination of low temperature and high loading rate (short HRT), the recorded diurnal variations in the rate of biogas production were either less pronounced or non-existent. The direct relationship between the rate of biogas production and the reactor temperature is supported by the known fact that microbial activity increases with temperature.

Under the operating temperature cycle of $15-25^{\circ} \mathrm{C}$, a steady gas production was achieved at the $25 \mathrm{~d}$ HRT only after a few days. At the $20 \mathrm{~d}$ HRT, a steady, but not rapid, decline in biogas production was observed after the $15^{\text {th }}$ day, following the initial rise in biogas production. At the $15 \mathrm{~d}$ HRT, the gas production declined unsteadily after the first 8 days until it completely stopped at the $28^{\text {th }}$ day. At the $10 \mathrm{~d}$ HRT, the gas production declined rapidly from the start, ceasing completely after the $5^{\text {th }}$ day. These results indicated that, the bioreactors did not operate satisfactorily at HRTs shorter than $25 \mathrm{~d}$ under the 15$25^{\circ} \mathrm{C}$ diurnal temperature cycle. The failure of the digesters at 15 and $10 \mathrm{~d}$ HRTs is due to the combined effects of the low cyclic diurnal temperature and high loading rate.

The biogas production observed at the $20-40^{\circ} \mathrm{C}$ diurnal temperature cycle was characterized by rapid rise in production followed by a rapid decline, the shorter the retention time, the more pronounced was the decline. This rapid rise in biogas production experienced at the beginning of each retention time can be attributed to the response of anaerobic bacteria to increased food supply as a result of the higher loading rate. Apparently, the acid forming bacteria responded faster to the increased loding rate. The accumulation of VFAs resulted in bacterial inhibition and, hence, reduction in biogas production. The higher rate of biogas production attained at a $20 \mathrm{~d}$ retention time indicated that at HRT longer than $20 \mathrm{~d}$, the reactors may have been underfed whereas at shorter retention times, the reactors may have been overloaded.

In this study, higher biogas production was obtained with the $20 \mathrm{~h}$ HRT. However, literature reports on effect of HRT on biogas production vary depending on the type of waste and system used. Hossain et al. (2009) noted that the biogas production increased in wastewaters as the HRT increased from $2-8 \mathrm{~h}$, but a 
further increase in the HRT from 8-14 h lead to a decrease in biogas production. Huang et al. (2011) reported that the higher biogas yields were achieved at lower HRTs (8-12 h) during wastewater treatment. Rico et al. (2011b) noted that as the HRT decreased from 20$12.5 \mathrm{~d}$, the biogas production increased from 0.66-1.21 $\mathrm{m}^{3}$ during the anaerobic digestion of dairy manure.

In this study, higher biogas production was observed with higher temperatures (within the temperature range studied). However the reports on the effect of temperature on biogas production vary. Hossain et al. (2009) reported that biogas production increased as the temperature increased from $30-40^{\circ} \mathrm{C}$ during wastewater treatment, but further increase in temperature from $40-45^{\circ} \mathrm{C}$ resulted in a decrease in biogas production. Wu et al. (2006) noted that the biogas production increased as the temperature increased from $20-40^{\circ} \mathrm{C}$ during anaerobic digestion of solid wastes and decreased with further increase in temperature to $45^{\circ} \mathrm{C}$ while a further increase to $55^{\circ} \mathrm{C}$ lead to a rapid increase in biogas production. Yu et al. (2002) reported that more biogas production was achieved at temperatures of $55^{\circ} \mathrm{C}$ during anaerobic digestion of wastewaters than those obtained at $37^{\circ} \mathrm{C}$. Banerjee and Biswas (2004) illustrated that during anaerobic digestion of wastes over the temperature range of $35-55^{\circ} \mathrm{C}$ maximum biogas production was achieved at $50^{\circ} \mathrm{C}$.

Biogas composition: Diurnal variations in gas composition were observed at the all HRTs under the $20-40^{\circ} \mathrm{C}$ diurnal temperature cycle. Similar variations were observed at the $20 \mathrm{~d}$ HRT under the $15-25^{\circ} \mathrm{C}$ diurnal temperature cycle. A better gas quality (higher methane content) was obtained at all HRTs under the $20-40^{\circ} \mathrm{C}$ diurnal temperature cycle. Higher methane content was also obtained at the 25 and 20 HRTs under $15-25^{\circ} \mathrm{C}$ diurnal temperature cycle. Rises in the methane content of biogas as a result of a decrease in the bioreactor temperature have been reported by other researchers (Peck et al., 1986). The increase in the quality of biogas is attributed to the raised solubility of carbon dioxide at the lower temperature cycle. Exceptions were observed with failed digesters. In stressed methanogenic systems, hydrogen production and volatile fatty acids (more reduced than acetic acid such as propionate, butyrate) have been reported to be produced in greater proportions (McInerney and Bryant, 1981).

The mean percentage of methane in the biogas ranged from $44.6-71.8 \%$, the carbon dioxide content ranged from $25.3-44.0 \%$ while the composition of the other gases (nitrogen, hydrogen sulphide) ranged from $0.1-11.8 \%$. Methane values reported in the literature ranged from 40-75\% (Converse et al., 1977; Hills and Stephens, 1980; Jeyanayagam and Collins, 1984; Lo and Liao, 1986; Peck et al., 1986). The observed values are, thus, within the range reported in the literature.

\section{CONCLUSION}

An experimental apparatus, including data acquisition and control system, was developed and used to investigate the effects of two diurnally cyclic temperature ranges $\left(20-40\right.$ and $\left.15-25^{\circ} \mathrm{C}\right)$ and four levels of hydraulic retention times $(25,20,15$ and $10 \mathrm{~d})$ on the performance of anaerobic reactors operated on screened dairy manure. The reactor temperature exhibited a lag relative to the chamber temperature. For the $20-40^{\circ} \mathrm{C}$ temperature cycle, the average lag at the maximum and minimum chamber temperatures and $4.37 \mathrm{~h}$, respectively. For the $15-25^{\circ} \mathrm{C}$ temperature cycle, the average lag at maximum and minimum chamber temperatures was 3.61 and $4.34 \mathrm{~h}$, respectively. The effluent solids content, total kjeldahl nitrogen and ammonium nitrogen concentrations were not adversely affected by the reactor diurnally cyclic temperature. The observed values of these parameters compared fairly well with the values obtained by other researchers. The effluent total solids, volatile suspended solids, TKN, $\mathrm{NH}_{4}-\mathrm{N}$ and methane content of the biogas diurnally cyclic patterns were out of phase with the diurnally cyclic pattern of the reactor temperature by about $12 \mathrm{~h}$ under most of the investigated operating conditions. The $\mathrm{pH}$ and the carbon dioxide content of the biogas all exhibited a diurnally cyclic pattern which was in phase with the reactor diurnally cyclic temperature. The reductions in total solids, total suspended solids, fixed suspended solids, total kjeldahl nitrogen and ammonium nitrogen and methane yield were all significantly affected by the diurnal temperature range and hydraulic retention time. The measured reactor $\mathrm{pH}$ followed a diurnally cyclic pattern which was in phase with the reactor temperature. At the $20-40^{\circ} \mathrm{C}$ temperature cycle the reactor $\mathrm{pH}$ ranged from 6.81-7.51 while VFA concentration ranged from 44.7$154.8 \mathrm{mg} \mathrm{L}^{-1}$. At the $15-25^{\circ} \mathrm{C}$ operating temperature cycle, the $\mathrm{pH}$ ranged from 6.30-6.60 while the VFA concentration ranged from $151.0-1187.0 \mathrm{mg} \mathrm{L}^{-1}$.

\section{ACKNOWLEDGEMENT}

This research was supported by the National Science and Engineering Research Council of Canada. 


\section{REFERENCES}

Al-Ghusain, I., M.F. Hamoda and M.A. El-Ghany, 2002. Nitrogen transformations during aerobic/anoxic sludge digestion. Bioresource Technol., 85: 147-154.

Alvarez, J.A., I. Ruiz and M. Soto, 2008. Anaerobic digesters as a pretreatment for constructed wetlands. Ecol. Eng., 33: 54-67. DOI: 10.1016/j.ecoleng.2008.02.001

APHA, 1985. Standard Methods for the Examination of Water and Wastewater. 16th Edn., American Public Health Association, Washington, D.C. ISBN: 0875531318

Andres, R.J., D.J. Fielding, G. Marland, T.A. Boden, N. Kumar and A.T. Kearney, 1999. Carbon dioxide emissions from fossil-fuel usage, Tellus, 51: 17511950. DOI: 10.1034/j.1600-0889.1999.t01-300002.x

Angelidaki, I and B.K. Ahring, 1994. Anaerobic thermophilic digestion of manure at different ammonia loads: Effect of temperature. Water Resources, 28: 727-731. DOI: 10.1016/00431354(94)90153-8

Appels, L., J. Degreve, B. Van der, J. Bruggen, V. Impe and R. Dewil, 2010. Influence of low temperature thermal pre-treatment on sludge solubilisation heavy metal release and anaerobic digestion. Bioresource Technol., 101: 5743-5748. DOI: 10.1016/j.biotech.2010.02.068

Blanchard, J.P. and T.A. Gill, 1987. Methane Production from Continuous Digestion of Farm Wastes. In: Global Bioconversion, Wise, I.D.L. (Eds.). C.R.C Press, Florida. ISBN: 0849345111

Banerjee, S. and G.K. Biswas, 2004. Studies on biomethanation of distillery wastes and its mathematical analysis. Chem. Eng. J., 102: 193201. DOI:10.1016/j.cej. 2004.05.006

Bradford, G.E., W.G. Pond and K.R. Pond, 2009. Animal Science and Production Technology. In: Adequate Food for All, Ponds, W.G., B.L. Nichols and D.L. Brown (Eds.). CRC Press, New York. ISBN: 9781420077537

Burke, D.A., 2001, Dairy Waste Anaerobic Digestion Handbook: Options for Recovering Beneficial Products From Dairy Manure. http://www.makingenergy.com

Chae, K.J., A. Jang, S.K. Yim and I.S. Kim, 2008. The effects of digestion temperature and temperature shock on the biogas yields from the mesophilic anaerobic digestion of swine manure. Bioresource Technol., 99 : 1-6. DOI: 10.1016/j.biotech.2006.11.063
Chen, Y.R. and D.L. Day, 1986. Effect of temperature change on stability of thermophilic fermentation of swine waste. Agric. Wastes, 16: 313-317. DOI: 10.1016/0141-4607(86)90061-2

Choorit, W. and P. Wisarnwan, 2007. Effect of temperature on the anaerobic digestion of palm oil mill effluent. Elect. J. Biotechnol., 10: 376-385. DOI: 10.2225/vol10-issue3-fulltext-7

Chu, C.Y., S.Y. Wu, P.C. Hsieh and C.Y. Lin, 2011. Biohydrogen production from immobilized cells and suspended sludge systems with condensed molasses fermentation solubles. Int. J. Hydrogen Energy, 36: 14078-14058. DOI:10.1016/j.ijhydene. 2011.04.101

Cleveland, C.J., 1995. The direct and indirect use of fossil fuels and electricity in USA agriculture, 1910-1990. Agric. Ecosystems Environ., 55: 111121. SSDI 0167-8809(95)00615-X

Colmenarejo, M.F., E. Sanchez, A. Bustos, G. Garcia and R. Borja, 2004. A pilot-scale study of total volatile fatty acids production by anaerobic fermentation of sewage in fixed-bed and suspended biomass reactors. Process Biochem., 39: 12571267. DOI: 10.1016/S0032-9592(03)00253-x

Converse, J.C., J.G. Zeikus, R.C. Graves and G.W. Evans, 1977. Anaerobic degradation of dairy manure under mesophilic and thermophilic temperatures. Trans. ASAE., 20: 336-340.

De la Rubia, M.A., M. Perez, L.I. Romero and D. Sales, 2002. Anaerobic mesophilic and thermophilic municipal sludge digestion. Chem. Biochem. Eng., 16: 119-124.

Dieu, T.T.M., 2009, Food Processing and Food Waste. In: Sustainability in the Food Industry, Baldwin, C.J. (Eds.). Wiley-Blackwell, Ames, Iowa. ISBN: 978-0813808468

Elango, D., M. Pulikesi, P. Baskaralingam, V. Ramamurthi and S. Sivanesan, 2007. Production of biogas from municipal solid waste with domestic sewage. J. Hazardous Mater., 141: 301-304. DOI: 10.1016/j.jhazmat.2006.07.003

Elefkiniotis P. and W.K. Oldham, 1994. Effect of HRT on Acidogenic digestion of primary sludge. J. Environ. Eng. ASCE, 120: 645-660.

El-Mashad, H.M., G. Zeeman, W.K.P. Van Loon, G.P.A. Bot and G. Lettinga, 2004. Effect of temperature and temperature fluctuation on thermophilic anaerobic digestion of cattle manure. Bioresource Technol., 95: 191-201. DOI: 10.1016/j.biotech.2003.07.013 
El-Mashad, H.M., W.K.P. Van Loon and G. Zeeman, 2003. A model of solar energy utilization in the anaerobic digestion of cattle manure. Biosystems Eng,. 84: 231-238. DOI: 10.1016/S15375110(02)00245-3

Ghaly, A.E. and E. Echiegu, 1993. Kinetics of a continuous flow no-mix anaerobic reactor. Energy Sources, 15: 433-449. DOI: 10.1080/00908319308909037

Ghaly, A.E., S.S. Sadaka and A. Huzzaa, 2000. Kinetics of an intermittent flow continuous mix anaerobic reactor. Energy Sources, 22: 525-542. DOI: $10.1080 / 00908310050013758$

Gustin, S. and R. Marinsek-Logar, 2011. Effect of pH, temperature and air flow rate on the continuous ammonia stripping of the anaerobic digestion effluent. Process Safety Environ. Protect., 89: 6166. DOI: 10.1016/j/psep.2010.11.001

Hills, D.J. and J.R. Stephens, 1980. Solar energy heating of dairy-manure anaerobic digesters. Agric. Wastes, 2: 103-118. DOI: 10.1016/01414607(80)90036-0

Hossain, M., N. Anantharaman and M. Das, 2009. Anaerobic biogas generation from sugar industry wastewaters in three-phase fluidized-bed bioreactor. Indian J. Chem. Technol., 16: 58-64.

Huang, Z., S.L. Ong and H.Y. Ng, 2011. Submerged anaerobic membrane bioreactor for low-strength wastewater treatment: Effect of HRT and SRT on treatment performance and membrane fouling. Water Res., 45: 705-713. DOI: 10.1016/j.watres.2010.08.035

Iannotti, E.L., J.H. Porter, J.R. Fischer and D.M. Sievers, 1979. Changes in swine manure during anaerobic digestion. Dev. Indus. Microbiol., 20: 519-529.

Izaurralde, R.C., 2009. Global Climate Change and Agriculture. In: Adequate Food for All, Ponds, W.G., B.L. Nichols and D.L. Brown, (Eds.). CRC Press, New York, ISBN: 9781420077537.

Jeyanayagam, S.S. and E.R. Collins, 1984. Weed seed survival in a dairy manure anaerobic digester. Trans. ASAE, 27: 1518-1523.

Kayranli, B. and A. Ugurlu, 2011. Effects of temperature and biomass concentration on the performance of anaerobic sequencing batch reactor treating low strength wastewater. Desalination. 278: 77-83. DOI: 10.1016/j.desal.2011.05.011

Kim, J.K., B.R. Oh, Y.N. Chun and S.W. Kim, 2006. Effects of temperature and hydraulic retention time on anaerobic digestion of food waste. J. Bioscience Bioeng., 102: 328-332. DOI: 10.1263/jbb.102.328
Legros, G., I. Havet, N. Bruce and S. Bonjour, 2009. The energy access situation in developing countries: A review focused on the least developed countries and sub Saharan Africa. http://content.undp.org

Lew, B., S. Tarre, M. Belavski and M. Green, 2004. UASB reactor for domestic wastewater treatment at low temperatures: A comparison between a classical UASB and hybrid UASB-filter reactor. Water Sci. Technol., 49: 295-301.

Lianhua, L., L. Dong, S. Yongming, M. Longlong and Y. Zhenhong et al., 2010. Effect of temperature and solid concentration on anaerobic digestion of rice straw in South China. Int. J. Hydrogen Energy. 35: 7261-7266. DOI: 10.1016/j.ijhydene.2010.03.074.

Lo, K.V. and P.H. Liao, 1986. Thermophilic anaerobic digestion of screened dairy manure using a twophase process. Energy Agric., 5: 249-255. DOI: 10.1016/0167-5826(86)90023-9

Macias-Corral, M., Z. Samani, A. Hanson, G. Smith, P. Funk and H. Yu, 2008. Anaerobic digestion of municipal solid waste and agricultural waste and the effect of co-digestion with dairy cow manure. Bioresource Technol., 99: 8288-8293. DOI: 10.1016/j.biortech. 2008.03.057

Maharaj, I. and P. Elefsiniotis, 2001. The role of HRT and low temperature on the acid-phase anaerobic digestion of municipal and industrial wastewaters. Bioresource Technol., 76: 191-197. PII: S 09608524 (00) 00128-0

Masse, D., Y. Gilbert and E. Topp, 2011. Pathogen removal in a farm-scale psychrophilic anaerobic digesters processing swine manure. Bioresources Technol., 102: 641-646. DOI: 10.1016/j.biotech.2010.08.020

McInerney, J.M. and M.P. Bryant, 1981. Basic Principles of Bioconversion in Anaerobic Digestion and Methanogenesis. In: Biomass Conversion Process for Energy and Fuel, Safar, S.S. and O.R. Zabarsky, (Eds.). Plenum Press. New York, ISBN: 0306406632.

Mogensen, L., J.E. Hermansen, N. Halberg, R. Dalgaard and J.C. Vis et al., 2009, Life Cycle Assessment across the Food Supply Chain. In: Sustainability in the Food Industry, Baldwin, C.J., (Eds.). Wiley-Blackwell, Ames, Iowa. ISBN: 9780813808468

Parkin, C.F. and W.F. Owen, 1986. Fundamentals of anaerobic digestion of wastewater sludges. J. Sanitary Eng. Division, Am. Soc. Civil Eng., 112: 867-920. DOI: 10.1061/(ASCE)07339372(1986)112:5(867) 
Peck, M.W., J.M. Skillon, F.R. Hawkes and D.L. Hawkes, 1986. Effect of temperature shock treatments on the stability of anaerobic digesters operated on separated cattle slurry. Water Res., 20: 453-462. DOI: 10.1016/0043-1354(86)90193-4

Pimentel, D. and M. Pimentel, 2008. Livestock Production and Energy Use. In: Food, Energy and Society: Pimentel, D. and M.H. Pimentel, (Eds.). CRC Press, New York, ISBN: 978-1-4200-4667-0.

Pimentel, D., B. Berger, D. Filberto, M. Newton and B. Wolfe et al., 2008a. Water Resources: Agricultural and Environmental Issues. In: Food, Energy and Society, Pimentel, D. and M.H. Pimentel (Eds.). CRC Press, New York, ISBN: 978-1-4200-4667-0

Pimentel, D., A.F. Warneke, W.S. Teel, K.A. Schwab and N.J. Simcox et al., 2008b. Biomass: Food versus Fuel. In: Food, Energy and Society, Pimentel, D. and M.H. Pimentel, (Eds.). CRC Press, New York, ISBN: 978-1-4200-4667-0.

Porpatham, E., A. Ramesh and B. Nagalingam, 2008. Investigation on the effect of concentration of methane in biogas when used as a fuel for a spark ignition engine. Fuel, 87: 1651-1659. DOI: 10.1016/j.fuel.2007.08.014

Pyle, L., 1978. Anaerobic Digestion: The Theoretical Options. In: Biogas Production in the Third World: A Multi-Disciplinary Review, Barnett, A., L. Pyle and S.K. Subramanian, (Eds.). International Development Research Centre, Ottawa. ISBN: 0889361622

Riano, B., B. Molinuevo and M.C. García-González, 2011. Potential for methane production from anaerobic co-digestion of swine manure with winery wastewater. Bioresources Technol., 102: 4131-4136. DOI:10.1016/j.biortech.2010.12.077

Ribas, M.M. and A.C. Barana, 2003. Start-up adjustment of a plug-flow digester for cassava wastewater (manipueira) treatment. Scientia Agric., 60: 223-229. DOI: 10.1590/S010390162003000200004

Rico, C., H. Garcia and J.L. Rico, 2011a. Physicalanaerobic-chemical process for treatment of dairy cattle manure. Bioresource Technol., 102: 21432150. DOI: $10.1016 /$ j.biortech. 2010.10.068
Rico, C., J.L. Rico, I. Tejero, N. Munoz and B. Gomez, 2011b. Anaerobic digestion of the liquid fraction of dairy manure in pilot plant for biogas production: Residual methane yield of digestate. Waste Manage., 31: 2167-2173. DOI: 10.1016/j.wasman.2011.04.018

Sooknah, R.D. and A.C. Wilkie, 2004. Nutrient remocal by floating aquatic macrophytes cultured in an aerobically digested flushed dairy manure wastewater. Ecological Eng., 22: 27-42. DOI: 10.1016/j.ecoleng.2004.01.004

Trazcinski, A.P. and D.C. Stuckey, 2011. Parameters affecting the stability of the digestate from a twostage anaerobic process treating the organic fraction of municipal solid waste. Waste Manage., 31: 1480-1487. DOI: 10.1016/j.wasman.2011.02.015

Umana, O., S. Nikolaeva, E. Sanchez, R. Borja and F. Raposo, 2008. Treatment of screened dairy manure by up flow anaerobic fixed bed reactors packed with waste tyre rubber and a combination of waste tyre rubber and zeolite: Effect of the hydraulic retention time. Bioresources Technol., 99: 74127417. DOI: 10.1016/j.biortech.2008.01.009

Wilkie, A.C., H.F. Castro, K.R. Cubinski, J.M. Owens and S.C. Yan, 2004. Fixed-film anaerobic digestion of slushed dairy manure after primary treatment: wastewater production and characterization. Biosystems Eng., 89: 457-471. DOI:10.1016/j.biosystemseng.2004. 09.002.

Wu, M., K. Sun and Y. Zhang, 2006. Influence of temperature fluctuation on thermophilic anaerobic digestion of municipal organic solid waste. J. Zhejiang University Sci., 7: 180-185.

Yu, H.Q., H.H.P. Fang and G.W. Gu, 2002. Comparative performance of mesophilic and thermophilic acidogenic upflow reactors. Process Biochem., 38: 447-454. 